\title{
Hippocampal theta sequences in REM sleep during spatial learning
}

Authors: Mark C. Zielinski ${ }^{1}$, Justin D. Shin ${ }^{1}$ and Shantanu P. Jadhav ${ }^{1,2 *}$

3

4 Affiliations:

$5 \quad{ }^{1}$ Graduate Program in Neuroscience

$6{ }^{2}$ Neuroscience Program, Department of Psychology, and Volen National Center for Complex

7 Systems

8 Brandeis University, Waltham, MA, 02453, USA

9

10 *Correspondence to: shantanu@brandeis.edu (S.P.J.)

11

12

13

14

15

16

17

18

19

20

21

22

23

24

25

26

27

28

29

30

31

32

33

34

35

36

37 


\section{ABSTRACT}

Rapid eye movement (REM) sleep is known to play a role in hippocampally-dependent memory, yet the activity and development of hippocampal neuronal ensembles during this state is not well understood. Here we investigated patterning of CA1 place cell activity by theta oscillations, a shared electrophysiological hallmark of both waking behavior and REM sleep, in male rats learning a spatial memory task. We report the existence of REM theta sequences, sequential reactivations of place cells in REM theta that parallel waking theta sequences. REM and wake theta sequences develop rapidly with experience, recapitulating behavioral sequences of compressed space in forward and reverse directions throughout learning. REM sleep exhibited a balance of forward and reverse sequences in contrast to predominantly forward wake theta sequences. Finally, we found that a CA1 neuronal population known to shift preferred theta phases in REM exhibited differential participation in wake and REM theta sequences. In particular, this phase-shifting population showed an increased contribution to REM theta sequence representations after behavioral performance asymptotes and the task is learned, supporting a previously hypothesized role in depotentiation. These findings suggest a role for REM associated theta sequences in state dependent memory functions of the hippocampal circuit, providing evidence that REM sleep is associated with sequence reactivation that can support consolidation of representations necessary for memory guided behavior. 


\section{INTRODUCTION}

Episodic memory guides behavior in the face of a continuously changing environment, integrating new information into existing representations of the external world or erasing old memories and creating new ones. The hippocampus is necessary for encoding and consolidation of memories from their labile form into stable long-term representations (Müller and Pilzecker, 1900; Dudai, 2004; Squire et al., 2015), with existing memory traces either updated, generalized, or forgotten (Alberini, 2005; Li et al., 2017; Izawa et al., 2019). Hippocampal place cells and their inputs support spatial and contextual memories (O'Keefe, 1976; Eichenbaum and Cohen, 2004; Dupret et al., 2010), by individually representing components of specific episodes (Moser et al., 2008) or sequences (Eichenbaum et al., 1999; Pastalkova et al., 2008; Tingley et al., 2018) in space and time. Sequential activation of place cells at fast timescales within single theta cycles and sharp-wave ripples can recapitulate this behavioral neural activity in a temporally compressed manner during both waking and offline states (Skaggs and McNaughton, 1996; Lee and Wilson, 2002; Karlsson and Frank, 2009; Jadhav et al., 2012; Drieu and Zugaro, 2019). These sequences are ideal for binding representations at a cellular timescale fast enough to trigger long term potentiation or depression (Mizumori et al., 1990; Bi and Poo, 1998), with waking and rest states thought to separate encoding and retrieval from consolidation and forgetting, respectively (Buzsáki, 1989; Mizuseki and Miyawaki, 2017; Poe, 2017; Samanta et al., 2020). and active sensation is patterned by the theta rhythm, a regular $6-12 \mathrm{~Hz}$ rhythm driven by the medial septum (Petsche et al., 1962) and seen in hippocampally associated areas during waking (Vanderwolf, 1969) and REM sleep (Jouvet, 1969; Buzsáki, 2002; Montgomery et al., 2008; Grosmark et al., 2012). The phase of spikes and ensemble firing relative to theta can directly influence neuronal plasticity across these areas (Huerta and Lisman, 1993, 1995; 


.

6

Hölscher et al., 1997; Orr et al., 2001; Griffin et al., 2004), and also induce bidirectional changes in memory and behavior (Pavlides et al., 1988; Hyman et al., 2003; Siegle and Wilson, 2014). The most well-known influence of this rhythm on individual place cells is the phenomenon of phase precession, where spikes shift their preferred firing relative to the phase of theta over the extent of a place field in an experience dependent manner (O'Keefe and Recce, 1993; Skaggs et al., 1996; Harris et al., 2002; Schmidt et al., 2009). Theta driven sequences emerge at the perspective of multiple place cells (Skaggs et al., 1996; Dragoi and Buzsáki, 2006; Foster and Wilson, 2007), are experience-dependent and separable from precession (Foster and Wilson, 2007; Feng et al., 2015; Silva et al., 2015), have been shown to map behavior in the forward and reverse directions (Cei et al., 2014; Wang et al., 2020), extend dynamically to goals (Gupta et al., 2012; Wikenheiser and Redish, 2015b), and serially represent options in deliberative decision making (Johnson and Redish, 2007; Gupta et al., 2012; Papale et al., 2016; Redish, 2016; Kay et al., 2020). Although theta oscillations are prominent in REM sleep, whether this theta in REM sleep organizes place cells into sequences in a similar manner as its waking counterpart is currently an open question.

REM sleep is associated with a unique neuromodulatory landscape and has been proposed to have roles in memory processes, specifically both consolidation and forgetting (Rasch and Born, 2013; Poe, 2017; Samanta et al., 2020). The theta oscillation itself undergoes a change in REM sleep, reversing its direction of flow through the hippocampal formation, from the subiculum to CA1 to CA3 (Jackson et al., 2014; Genzel et al., 2015). REM also induces transient changes in synapse maintenance and formation, cellular excitability, and ensemble synchrony (Montgomery et al., 2008; Grosmark et al., 2012; Li et al., 2017). Superficial and deep CA1 neurons are modulated by REM differently, with deep CA1 cells shifting their preferred theta phases from wake to REM sleep (Poe et al., 2000; Mizuseki et al., 2011; Valero et al., 2015; Wang et al., 2020). Reverberations of waking activity in REM sleep, at the same 
timescale as behavioral activity, have also been reported (Louie and Wilson, 2001; Peyrache et

117 al., 2015), though the development and detailed properties of this reactivation is unknown.

118 Experience and novelty dependent changes in REM theta are thought to occur from these and 119 other lines of evidence, but recent findings have been mixed (Poe et al., 2000; Mizuseki et al., 120 2011). Further, despite the similar network states in REM and wake, and the role that theta

121 plays in episodic memory, little is still known about the existence of compressed hippocampal

122 theta sequences in REM, their functional properties and development, and their potential role in 123 behavior. We therefore investigated this question using continuous hippocampal CA1 ensemble 124 recordings across interleaved behavior and rest sessions in rats learning a spatial alteration 125 task in a single day.

RESULTS

Trajectory representation and REM sleep during single-day acquisition of a W-track spatial alternation behavior

In order to address the potential role that theta associated place cell sequences have in REM sleep, we monitored hippocampal ensembles in vivo throughout single-day acquisition of a W-track spatial alternation task known to require working memory (Figure 1A,B) (Jadhav et al., 2012; Maharjan et al., 2018; Shin et al., 2019). The W-track continuous alternation task requires

137 animals to visit reward ports situated at the end of the three track arms in a specific behavioral 138 sequence. Correct inbound or outbound trials are rewarded in the following manner: starting 139 from the center arm, the animal must visit an outer arm reward well (outbound trajectory), then 140 return from the outer arm well to the center arm well (inbound trajectory) to consume reward. All 141 subsequent outbound trials require the animal to recall the previous outer arm visit, and visit the 
142

143

144

145

146

148

149

150

151

152

153

154

155

156

157

158

159

160

161

162

163

164

165

166

167

opposite outer arm well that was not previously chosen. This sequence then continues, with center arm visits interleaved with alternating outer arm visits (Figure 1A, left). Run sessions consist of $15-20$ minutes of exposure to this track, followed by a $\sim 30$ min rest session in a familiar sleep box (Figure 1A, right).

Custom adjustable microdrive arrays were used to target tetrodes to dorsal CA1 regions (see Methods, Figure 1C). Electrophysiological recordings monitored activity of hippocampal ensembles throughout the entirety of the experiment over task acquisition (5.5-6.5 hours). A behavioral epoch consisted of one run session, followed by one sleep session, for a total of 8 behavioral epochs (Figure 1B, an initial sleep session prior to track exposure was not used for this analysis). Place cell templates in a given run session were used for subsequent decoding in the following sleep session. All animals exhibited rapid learning of the task (Figure 1F), and the proportion of correct overall trials, and therefore learning, was asymptotic by behavioral epoch 5 (Kruskal-Wallis with Tukey-Kramer correction for multiple comparisons between the 8 behavioral epochs; $X^{2}(7)=31.81$, comparison between epochs 1 and 5 significantly different, $p=$ 0.0096; all comparisons between epochs $5-8$ were not significantly different, $p>0.76$ ). This experimental design thus allowed for investigation of CA1 dynamics in waking and sleep states over the course of learning, from initial acquisition to later correct performance (mean \pm SEM number of hippocampal place cells per animal epoch; wake, $47 \pm 1.5$ cells; sleep, $40 \pm 1.2$ cells).

REM sleep in rodents is fragmented, with changes in LFP rhythms during sleep reminiscent of wake, namely increase in theta $(6-12 \mathrm{~Hz})$ power and decrease in delta $(1-4 \mathrm{~Hz})$ power (Twyver, 1969; Rasch and Born, 2013). Using established methods for REM detection ((Grosmark et al., 2012; Kay et al., 2016; Tang et al., 2017), see Methods and Figure 1D), namely immobility, lack of ripple power corresponding to sharp wave ripple events, and high 
168

169

170

171

172

173

174

175

176

177

178

179

180

181

182

183

184

185

186

187

188

189

190

theta to delta ratio, the fraction of REM sleep during rest sessions was consistent across all behavioral sessions (Figure 1E, Kruskal-Wallis with Tukey-Kramer correction for multiple comparisons between the 8 behavioral epochs; $X^{2}(7)=3.69, p=0.8152$, all comparisons $p>$ 0.8050), in agreement with prior literature (Twyver, 1969; Mendelson and Bergmann, 1999).

CA1 place cells are direction and trajectory selective, with distinct spatial encoding of all 4 trajectories (outbound left, outbound right, inbound left, and inbound right) in the W-track task (Shin et al., 2019). Figure 1G shows normalized firing rates of all place cells for a single animal and epoch, sorted according to place field peak in the respective trajectory. These ensembles are directionally selective from the onset of behavior and direction selectivity develops rapidly with experience, as previously reported (Frank et al., 2004; Foster and Wilson, 2006; Shin et al., 2019; Xu et al., 2019). Further, as we have previously shown (Shin et al., 2019), trajectory specific sequences of place cell ensembles were unique and differentiable from one another for each animal and behavioral epoch (Figure 1H, permutation test, all comparisons between real and shuffled data; $p<0.01$ ), allowing for unambiguous detection, identification, and tracking of potential forward and reverse sequences throughout behavior. The stability of REM sleep fraction during single day learning, in conjunction with monitoring of hippocampal ensembles, enabled us to examine the development of hippocampal ensemble activity patterns in waking and REM states.

\section{Theta properties and parameters in wake and REM sleep}

We first examined theta-phase associated properties of place cells in the two states, wake and REM Sleep. In particular, since reversal of preferred theta phase in REM sleep is known to occur for a subset for place cells (Poe et al., 2000; Mizuseki et al., 2011), we examined the prevalence of REM theta phase reversal during spatial alternation learning. We 
194

195

196

197

198

199

200

201

202

203

204

205

206

207

208

209

210

211

212

213

214

215

216

217

218

219

found large fractions of neurons significantly phase locked to theta (Rayleigh z test for nonuniformity $p<0.05$ ) in wake and REM sessions (mean \pm SEM phase locked cells per animal epoch; Wake $61 \% \pm 8.9 \%$ (30 \pm 4 cells $)$; REM sleep $37 \% \pm 5.3 \%(15 \pm 2$ cells $)$ ). The distributions of mean preferred phases in wake and REM had a small but significant difference (Figure 2A, Kuiper two-sample test; the two distributions for preferred phases of the phaselocked neurons are significantly different, $\mathrm{T}($ Kuiper $)=1.3 \mathrm{e} 04, \mathrm{p}=0.005)$, in agreement with previous literature (Poe et al., 2000; Mizuseki et al., 2011). To investigate whether a subset of hippocampal neurons in this task also reversed their preferred phases, we analyzed place cells which had significant phase-locking in both wake and REM sleep (per animal mean \pm SEM; $30 \pm$ 5 cells). We found that a fraction of place cells shifted their preferred phases in REM sleep

(Figure 2B; over all animals and behavioral epochs, $30 \% \pm 2.3 \%$ (9 \pm 1 cells), mean \pm SEM), using criteria (see Methods) similar to previously established guidelines delineating shifting cells from non-shifting cells (Mizuseki et al., 2011). Individual examples of two cells in Figure 2C show these phase preference shifts between Wake and REM.

In light of this notable fraction, we next examined theta phase-locking and phase-shifting throughout the duration of learning. Figure 2D shows the preferred theta phases over all epochs for shifting and non-shifting cells, with interleaved wake and REM sleep sessions. Mean preferred theta phases split by shifting and non-shifting cells show a clear transition from wake to REM sleep for the shifting cells for all epochs. In wake, preferred theta phases are statistically similar between shifting and non-shifting cells (Watson-Williams test, $p=0.52$ ), in agreement with previous findings (Mizuseki et al., 2011) (Figure 2E, left). Preferred phases in REM are significantly different between the two populations (Watson Williams test, $p<1$ e-99) (Figure 2E, right). This shifting effect across all epochs is summarized as polar plots in Figure 2F for wake (left) and REM (right). The number of phase-shifting cells did not change significantly across epochs during single day learning (there was no statistically significant difference in proportion 
220

221

222

223

224

225

226

227

228

229

230

231

232

233

234

235

236

237

238

239

240

241

242

243

244

245

of wake and REM phase shifting cells over epochs, $X 2(7)=12.69, p=0.94 ; X 2$ test of

independence). We also examined properties of theta oscillations in the two states and found an overall similarity in theta frequency content $(6-12 \mathrm{~Hz}$, see Methods) across wake and REM in all animals (Figure 2G), with mean theta frequencies similar across the two behavioral states

throughout the course of behavior (Figure $\mathbf{2 H}$; there was no statistically significant difference between wake and REM theta frequencies, $X 2(1)=0.6847, p=0.4080$. Friedman Test).

\section{Detecting theta sequences in wake and REM sleep}

Next, in order to assess the existence, content, and evolution of theta sequences, we used established methods to detect candidate periods of theta cycles during behavior and REM (see High-theta segmentation and REM state identification, and Theta cycles and decoding in

Methods for details), and then used a Bayesian decoding approach to identify sequential reactivation events (Zhang et al., 1998; Davidson et al., 2009; Feng et al., 2015). Theta cycles, and therefore sequences, were segmented from trough to trough in waking behavior (Johnson and Redish; Gupta et al., 2012; Feng et al., 2015; Wu et al., 2017). Since there is a prevalence of phase reversing cells in REM sleep which preferentially fire during theta trough (Figure 2), as well as the known reversal of activity flow of the theta rhythm in REM (Jackson et al., 2014; Genzel et al., 2015), we segmented theta cycles from peak to peak in REM sleep. This also allowed us to assess the contribution of phase-reversing cells to potential theta sequences in REM. Figure S1A shows LFP, theta phase, spikes, animal position, and decoding for a 0.5 second period of behavior on the W-track task, with decoded track position for each of the four trajectories. Figure S1B shows the same for an equivalent segment of REM sleep, using place cell templates from the preceding run session.

Sequential structure of reconstructed position during reactivation is evaluated primarily by a trajectory score measure, which assesses a decoded Bayesian posterior for replay-like 
structure by finding the optimal virtual slope and intercept that maximizes an objective function -

247 in this case, the total amount of decoded probability captured within the boundaries of a linear fit

248 ((Kloosterman et al., 2014); see Methods; decoded trajectory seen as a superimposed dotted

249 white line in Figure S1A,B and examples in Figure 3). This captured probability, normalized by

250 the total maximum theoretical probability that could be captured, is computed as the trajectory

251 score, as previously described (Kloosterman et al., 2014; Drieu et al., 2018). We saw a

252 substantial proportion of decodes with sequential, linear structure in both wake and REM sleep

253 using this ensemble approach to delineate theta sequence events from non-sequence events

254 (Figure 3).

Previous literature has advocated for this ensemble approach to identify sequential reactivation events (Foster, 2017). We therefore used a rigorous combination of trajectory scores, shuffles, and other complementary measures to separate statistically significant decodes from candidate events and to reduce the risk of false positives, and applied the same method to both wake and REM states. Line fitting scores were overall higher in wake as compared to REM sleep (Figure S1C, wake and REM sleep distributions are different, $p<1 e-$ 99, rank-sum test), indicating the higher fidelity of decoded sequential positions and putative theta sequences in wake. Fitted trajectory slopes (Figure S1D) were significantly higher in REM decodes than in wake (wake and REM sleep distributions are different, $p<3.41$ e-59, rank-sum test), indicating higher virtual decode velocities, which were also mirrored in the absolute decode distances elapsed in these decodes (Figure S1E) and their differences (wake and REM

267 sleep distributions are different, $p<1.46 e-47$, rank-sum test). Though recent work has provided 268 a fascinating possible role for stationary representations of space in theta decodes (Muessig et 269 al., 2019), we imposed virtual decode velocity cutoffs of greater than $1 \mathrm{~m} / \mathrm{s}$ and less than $10 \mathrm{~m} / \mathrm{s}$ 270 to only analyze non-stationary decodes, or decodes with velocities approaching those reported 271 of sharp-wave ripple replay to avoid potential, though unlikely false negatives. 
Similar to reported methods from prior studies analyzing theta sequences (Zhang et al., 1998; Davidson et al., 2009; Karlsson and Frank, 2009; Gupta et al., 2012; Pfeiffer and Foster, 275 2013), we employed two shuffle procedures on decoded spatial posteriors, permuting both 276 posterior time bin order (Figure S1F) or circularly permuting spatial bins within each time bin

277 (Figure S1G) 1000 times, computing optimal line fitting trajectories for each permutation. The 278 time bin shuffle or space shuffle scores were then computed as the percentile of the real. We 279 imposed a $99^{\text {th }}$ percentile cutoff to ensure that putative theta sequences had scores distinguishable from those possible by chance alone ( $p<0.01$ criterion). Wake and REM sequences had small but significant differences in shuffle scores (Figures S1F-G; wake and REM sleep distributions are different, both $p<3.28$ e-33, rank-sum test). that impose both regularity and continuity of decoded sequential position, limiting both jump decode by limiting the total number of silent time bins within the probability containing portion of a decode posterior.

In cases where more than one trajectory template of a putative sequence passed these statistical criteria, we chose the trajectory template that had the highest real trajectory score, for

295 both wake and REM sleep. Using this rigorous ensemble of statistical methods applied

296 equivalently to both states, we investigated the properties of detected theta sequences in wake 297 and REM sleep. 


\section{REM theta sequences mirror waking theta sequences}

With a principled way of delineating theta sequences from putative spatial decodes for both wake and REM, we examined the representation of trajectories, directionality, and other properties of these sequences. Figure 3A-G show six example decoded theta sequences, with Figure 3A-C showing wake decodes, and Figure 3D-F showing REM decodes. Additional examples are shown in Figure S2. Each example shows place cell activity (lower left) during a single cycle of theta oscillation (broadband/theta LFP in upper left) for a candidate event.

306 Templates for all four trajectories are shown as linearized place fields sorted by peak locations on the detected theta sequence trajectory (bottom right), with the chosen/detected trajectory highlighted in red. Bayesian reconstructions of position from place cell firing in these cycles

We quantified the prevalence of theta sequences and their properties in wake and REM over the course of learning. The total proportion of significantly decoded theta sequences was significantly different for wake and REM sessions, with $\sim 14-17 \%$ for wake and $\sim 8-11 \%$ for REM

320 (Figure 3G; There was a statistically significant difference between wake and REM theta 321 proportions, $X 2(1)=25.96, p=3.48 \mathrm{e}-07$. Friedman Test.). A majority of these sequences were

322 in the forward direction for wake, while the proportion of forward decodes in REM were 323 balanced with those in the reverse direction, Figure $3 \mathbf{H}$ (proportion of forward theta sequences 
324 for wake vs. REM, $X 2(1)=22.62, p=1.98 e-6$. Friedman Test). We also saw a consistent

325 difference in mean absolute decode slope between wake and REM (Figure 3I; there was a

326 statistically significant difference between wake and REM theta sequence slopes, $X 2(1)=40.21$,

$327 p=2.29 \mathrm{e}-10$. Friedman Test.) and decode extent (Figure 3J; wake vs. REM theta sequence

328 extents, $X 2(1)=40.21, p=2.29 e-10$. Friedman Test).

Theta sequences in wake and REM sleep propagate differentially in forward and reverse directions

In finding the optimal theta decode, we allowed any given trajectory or slope, and found

that a large fraction of reverse theta decodes were chosen as being the most likely decoded sequences, in contrast to predominantly forward sequences in wake (examples in Figure 3, future positions respectively during behavior (Wang et al., 2020).

In order to further quantify the robustness of this sequential decoding and to ascertain forward and reverse sequences, we employed an additional quantification metric known as the quadrant ratio, which uses the shape of the decoded posterior over time to capture the magnitude and direction of a decoded sequence. Here, the quadrant ratio imposes a second,

347 more stringent criterion for each individual sequence, to further assess the statistical validity of 348 decoded sequences with significant trajectory scores. To examine decoded waking sequences 349 assessed by quadrant scores, average theta sequence decodes centered on animal position 
were generated over the course of behavior for both forward and reverse sequences (top and bottom in Figure 4A). These sequences show average structured sequential representations per epoch, with average LFP waveforms segmented by theta overlaid in white. Theta waveform asymmetry did not change significantly over learning for either wake or REM (Figure S3A-D).

Each individual sequence centered on current animal position was assessed for quadrant score significance using spatial and time bin shuffles. Figures 4B-C show significance values from permutation tests comparing the probability of true quadrant scores relative to their shuffled counterparts (see Methods) for all individual wake theta sequences over learning in the forward (Figure 4B) and reverse (Figure 4C) directions, respectively. Shuffled distributions for each decode consist of random circular permutations of space in each decode time bin (space shuffle), and randomly permuting time bins (time shuffle). The permutation tests show that quadrant ratios develop rapidly for forward sequences in wake, especially in the second behavior epoch. Quadrant score permutation tests for large majority of forward theta sequences aligned by current animal position were clearly significant relative to shuffled distributions (Figure 4B-C; $p=0.05$ is indicated by the dotted line, and $p$-values for median scores indicated by thick horizontal line overlaid on shuffle distributions. Note that the majority of $p$-value distributions lie below the $p=0.05$ threshold for forward sequences from epoch 2 onward), indicating rapid formation of significant forward theta sequences early in behavior relative to shuffled controls. In contrast, only a small subset of individual reverse sequences in wake are significant for the quadrant score statistic (Figure 4C; with $p=0.05$ indicated as a dotted line). Reverse theta sequences initially comprised a smaller proportion of wake theta sequences as assessed by the trajectory score metric (Figure $3 \mathbf{H}$ ), and this quadrant ratio metric further suggests that significant reverse sequences are not very prevalent as compared to forward sequences during behavior. 
We performed a similar quantification (Figure 4D-F) for REM sleep. Average theta

sequences relative to current animal position and corresponding quadrant ratio scores are only valid for wake theta sequences. Since REM theta sequences were detected using waking trajectory templates and do not correspond to current animal position on the maze trajectories, this necessitated centering of decodes at the midpoint for quadrant score computation in REM (Figure 4). REM theta sequences were centered around decode midpoints, and again split into forward and reverse sequences. Quadrant score permutation tests were significant relative to both shuffle controls throughout learning for majority of REM sequences in either direction, forward and reverse. This confirms results from Figure 3 that REM theta sequences develop rapidly and exhibit compressed sequential reactivation of waking spatial behavior in forward and reverse directions. Since centering by decode midpoint rather than animal position results in a more structured quadrant score by design, a stringent evaluation of the quadrant score statistic for REM sequences similar to wake sequences is not feasible. An equivalent comparison for wake theta sequences centered by decode midpoint is shown in Figure S4A-C.

\section{Theta sequence content and participation of phase reversing cell ensembles}

We next wanted to determine whether and how the content of these theta sequences changes over time, and whether phase shifting cells (Figure 2) had any role in these sequences. Given the known difference in afferent connections between superficial and deep CA1 neurons, and their phase reversal in REM sleep (Mizuseki et al., 2011; Valero et al., 2015; Oliva et al., 2016b, a), we quantified the proportion of sequences with phase shifting neurons (termed "shifter sequences") relative to those without phase shifting neurons (termed "nonshifter sequences", sequences with a threshold of $25 \%$ or more shifting cells were considered to be shifting cell predominant; qualitatively similar results were seen with a threshold of $50 \%$, see

\section{Methods).}


First, we found that wake sequences overall have relatively even proportions of shifting and non-shifting sequences seen throughout behavior, whereas REM sequences comprise overall more non-shifting sequences (Figure 5A; there was a statistically significant difference between the proportion of wake and REM shifter and non-shifter sequences, $x 2(1)=8.16, p=$ 0.0043, Friedman Test). In order to summarize the content of these sequences and ascertain the contribution of the phase-shifting cell participation, we generated quiver plots showing the extent of theta sequence decodes (Figure 5B-C) in the forward direction for wake (top), and forward and reverse directions for REM sleep (bottom). Examples of quiver plots for illustrative individual sessions in early and late learning epochs are shown in Figure 5B-C respectively, and distributions of decoded positions attributed to shifter vs. non-shifter sequences are shown as histograms of spatial bin counts in the topmost row in Figure 5B-C. Ensembles which had

414 more than $25 \%$ of phase shifting cells participating are in red ("shifter sequences", see

415 Methods; qualitatively similar results were obtained using a 50\% threshold). For REM sequences (Figure 5B-C, bottom), note the lack of phase-shifting cell sequences (non-shifter sequences) in the early epoch, and the emergence of shifter sequences in the late epoch.

We also examined spatial coverage of these decoded sequences across learning, quantified as histograms of spatial bin counts (summarized in Figure 5D). Decoded spatial

421 coverage shows increase of track representation by shifter sequences in REM over learning

422 (Figure 5D,E). We thus found that in REM, the contribution of sequences comprising phase-

423 reversing cells to decoded locations on track, relative to sequences with predominantly non-

424 phase reversing cells, increases over learning (Figure 5E; there was no statistically significant 425 difference in the normalized bin decode proportion of shifter vs. non-shifter for wake sequences 426 over epochs, $\mathrm{X} 2(7)=12.98, \mathrm{p}=0.0726$. Kruskal-Wallis test; there was a statistically significant 
427

428

429

430

431

432

433

434

435

436

437

438

439

440

441

442

443

444

445

446

447

448

449

450

451

452

difference in the normalized bin decode proportion of shifter vs. non-shifter for REM sequences over epochs, $\mathrm{X} 2(7)=23.2, \mathrm{p}=0.0016$. Kruskal-Wallis test).

\section{DISCUSSION}

Our results establish the existence of theta sequences in REM sleep. We show that during spatial learning, REM theta sequences recapitulate time-compressed behavioral activity in the forward and reverse directions. REM theta sequences mirror the evolution and content of theta sequences in waking behavior at the trough of the theta cycle, with REM sleep exhibiting a balance of forward and reverse sequences in contrast to predominantly forward wake theta sequences. Finally, REM phase shifting and non-shifting cells, i.e. putative superficial and deep CA1 cells (Mizuseki et al., 2011; Poe, 2017), differentially participate in this evolution over behavior. Phase-shifting cells showed an increase in contribution to REM theta sequence representation after acquisition of learning. The existence of theta sequences in REM sleep, their development over learning of a memory-guided behavior, and the increased participation of a subset of phase reversing CA1 neurons in these sequential representations after learning, together point to REM theta-mediated sequences as another potential mechanism in hippocampally driven memory processes.

We find two notable differences in REM and wake theta sequences - balance of forward and reverse sequences, and differential participation of phase-shifting and non-phase shifting cells. The difference in the balance of forward and reverse theta sequences in waking and REM sleep can potentially be explained by the fact that waking circuits may be biased or shaped by incoming sensory input from entorhinal cortex and concurrent knowledge about the task structure from CA3. Forward theta sequences predominate in wake, and several previous studies have reported their role in deliberation, heading and goal representation (Johnson and 
453

454

455

456

457

458

459

460

461

462

463

464

465

466

467

468

469

470

471

472

473

474

475

476

477

478

Redish, 2007; Gupta et al., 2012; Cei et al., 2014; Wikenheiser and Redish, 2015a; Papale et al., 2016; Redish, 2016). Reverse theta sequences during wake have been reported (Wikenheiser and Redish, 2013; Zheng et al., 2016; Drieu and Zugaro, 2019; Schmidt et al., 2019), but to our knowledge, their role in behavior is not clear. A recent study used high-density hippocampal recordings to report the existence of reverse and forward theta sequences within individual cycles representing past and future positions respectively during behavior (Wang et al., 2020). This study also reported that REM phase-shifting cells are more likely to participate in sharp-wave ripple (SWR) associated reverse replay in sleep, suggesting a link to SWRmediated consolidation.

Here, we observed a balance of forward and reverse theta sequences in REM that recapitulate recent experience in a time-compressed manner. The neuromodulatory landscape of REM shares an elevation of cholinergic tone with waking behavior, thought to be driven in part by the medial septum and other circuitry (Müller and Remy, 2017). A notable difference between these states are low levels of noradrenergic and serotonergic signaling, along with bursts of dopaminergic signaling in the hippocampus during REM sleep (Mizuseki and Miyawaki, 2017; Poe, 2017; Samanta et al., 2020). Interestingly, activity flow of the theta rhythm has been reported to reverse in REM via GABAergic mechanisms, propagating from the subiculum to CA3 exclusively during the neuromodulatory environment of REM sleep. Reverse theta sequences may thus be enabled by the unique neuromodulatory tone during REM sleep (Jackson et al., 2014; Genzel et al., 2015), and possibly play specific roles in memory. learning also suggests a specific role in memory processes. REM phase-shifting and non-phase shifting cells are known to be anatomically differentiated. The radial axis within CA1 is delineated in part by the differences in afferent connectivity, both external to the hippocampal 
479

480

481

482

483

484

485

486

487

488

489

490

491

492

493

494

495

496

497

498

499

500

501

502

503

504

circuit via MEC and LEC input to differing apical dendrites (Li et al., 2017; Masurkar et al., 2017), and within the hippocampal circuit, via CA2 and CA3 input to deep and superficial basal dendrites, respectively (Valero et al., 2015; Oliva et al., 2016b, a). This in turn informs their functional properties; place fields from superficial cells are less numerous and more stable, while deep cells have more place fields, which are flexible and encode task features, landmarks, and rewards (Mizuseki et al., 2011; Danielson et al., 2016; Geiller et al., 2017).

We observed differential prevalence of phase-shifting cells in REM vs. wake theta sequences, and increased participation of these phase-shifting cells in REM sequence representations after learning had occurred. This change in phase-shifting cell participation was selectively seen only in REM sequences and not wake sequences. Although there is a possibility that more shifting-cell theta sequences were simply detected later in learning based on the statistical methods employed, this is unlikely since the number of phase-shifting cells did not change across epochs, and the same statistical criteria were employed throughout learning for both wake add REM sequences. Rather, the emergent participation of phase-shifting cells in theta sequences suggests a potential role in memory processes, with a likely function being forgetting through depotentiation, as hypothesized previously (Poe, 2017).

\section{Since firing of place cells at the theta trough is known to induce LTD at synapses} (Huerta and Lisman, 1993, 1995; Hölscher et al., 1997; Orr et al., 2001; Griffin et al., 2004), it has been hypothesized that firing of phase-shifting cells at theta troughs in REM sleep can support depotentiation for circuit remodeling and forgetting (Poe, 2017). Such depotentiation may support new learning by weakening old associations and subsequently enabling new associations, or have a role in sparsening the contextual ensemble code for an environment as a means to generating a simplified code from an initial over-representation of environmental variables (Rasch and Born, 2013; Poe, 2017; Samanta et al., 2020). Our finding of REM 
505

506

507

508

509

510

511

512

513

514

515

516

517

518

519

520

521

522

523

524

525

526

527

528

529

530

531

532

533

534

535

536

537 sequences at theta troughs and the increased participation of phase-shifting cells in REM sequences after learning is suggestive of a possible mechanism for ensemble pattern sparsening through depotentiation of phase-shifting cells in REM. We speculate that if phaseshifting hippocampal neurons contribute to new and labile episodic representations, then the REM neuromodulatory environment can allow for de-potentiation and sparsening of newly formed synapses through theta sequences propagating in the forward and reverse direction. Labile representations mediated by REM phase-shifting hippocampal neurons can preferentially fire at the theta trough to eliminate any non-essential patterns of activity, leading to circuit remodeling and simplification of the contextual ensemble code. Theta sequences in REM can thus allow for exploration of novel recombination of experience with forward and reverse sequences, and simultaneous de-potentiation of selective sequences. Since phase-shifting cells preferentially participate in SWR reverse replay (Wang et al., 2020), the possibility of interactions between reverse theta sequences in REM and reverse SWR replay sequences in NREM sleep also cannot be ruled out. These hypotheses can be tested in future studies that examine the role of phase-shifting cells in learning multiple novel experiences, including reversal learning (Poe, 2017). Our findings thus support a potential role of REM sleep in memory formation, and establish the existence of bi-directional REM theta sequences as an integral component of the machinery involved in memory processes in sleep. 


\section{ACKNOWLEDGEMENTS}

540

541 This work was supported by NIH Grant R01 MH112661 to SPJ. Analyses were performed

542 using Brandeis University's High Performance Computing Cluster which is partially funded

543 by DMR-MRSEC 1420382.

544

545

546

AUTHOR CONTRIBUTIONS

547 M.C.Z. designed and performed all data analyses; J.D.S. performed experiments; S.P.J.

548 conceived the study and supervised all aspects of the work; M.C.Z. and S.P.J. wrote the 549 manuscript with input from all authors.

550

DECLARATION OF INTERESTS:

552 The authors declare no competing interests.

553

554

555

556

557

558

559

560

561

562

563

564

565

566

567

568

569

570

571

572 
573

574

575

576

577

578

579

580

581

582

583

584

585

586

587

588

589

590

591

592

593

594

595

596

597

598

\section{METHODS}

\section{Animals and experimental design}

Six adult male Long Evans rats (450-550 g, 4-6 months, RRID: RGD_2308852) were used in this study. Data from four of these animals during behavior sessions has been reported previously (Shin et al., 2019). All procedures were conducted in accordance with the guidelines of the US National Institutes of Health and approved by the Institutional Animal Care and Use Committee at Brandeis University. Animals were housed individually and kept on a standard $12 \mathrm{~h} / 12 \mathrm{~h}$ light-dark cycle with ad libitum food and water available. Daily handling and habituation took place prior to training and experimental protocols, with all protocols carried out during animals' light cycles. As previously described (Jadhav et al., 2016; Tang et al., 2017; Shin et al., 2019), animals were food deprived to $85-90 \%$ of their stable ad libitum weight and trained to seek liquid food reward (evaporated milk) alternating between elevated linear track ends (80 $\mathrm{cm}, 7 \mathrm{~cm}$ wide track sections). Animals were also exposed and habituated to an enclosed and opaque elevated rest box during this training period over multiple days. After animals reached a criterion level during the linear track protocol (50 rewards in 15-20 minute linear track sessions), they were taken off food restriction and chronically implanted with a multi-tetrode drive (see Surgical procedures, euthanasia, and histology). Following recovery, animals were again food deprived to $85-90 \%$ of their ad libitum weight and re-habituated to the linear track task. Animals were then exposed to the full novel W-track continuous alternation behavioral task during the recording day (see Behavioral task), with electrodes positioned appropriately in the stratum pyramidale layer on the previous day.

\section{Behavioral task}


Rats performed a continuous W-track spatial alternation task, as previously described

600

601

602

603

604

605

606

607

608

609

610

611

612

613

614

615

616

617

618

619

620

621

622

623

624

(Jadhav et al., 2012; Jadhav et al., 2016; Tang et al., 2017; Maharjan et al., 2018; Shin et al., 2019). An experimental day consisted of multiple interleaved behavioral sessions (8 sessions) on the $\mathrm{W}$-track and inactive periods in the elevated rest box, consisting of 15-20 min run sessions and 30-40 min rest sessions, respectively (Figure 1A, B). The W-track ( 80 x $80 \mathrm{~cm})$ consists of elevated track sections ( $7 \mathrm{~cm}$ wide) with reward wells situated at the end of each of the three arms. The three arms were connected with two short sections ( $40 \mathrm{~cm}$ long) for a total of $\sim 200 \mathrm{~cm}$ for a behavioral trajectory from the center arm reward well, to the outer arm reward well. Calibrated evaporated milk rewards were delivered automatically via infrared detectors integrated in reward wells for correct trials. Rats were tasked with learning a continuous spatial alternation strategy (starting from the center arm), alternating visits to either side well (outbound component) and the center well (inbound component). Incorrect alternations (visiting the same side well in consecutive outbound components - outbound error), incorrect side-to-side well visits (without visiting the center arm - inbound error), or perseverations (repeated visits to the same well just visited) were not rewarded. Only correct trials were used for further analysis.

\section{Surgical procedures, euthanasia, and histology}

Surgical implantation procedures were as previously described (Jadhav et al., 2012; Jadhav et al., 2016; Tang et al., 2017; Shin et al., 2019), and post-operative analgesia administered for several days post implantation, along with free food and water. Briefly, each rat was surgically implanted with a 3D printed microdrive containing 30 independently moveable tetrodes, with 15 tetrodes targeting right dorsal hippocampus (-3.6 mm AP and $2.2 \mathrm{~mm} \mathrm{ML)}$ and 15 tetrodes targeting right PFC (+3.0 mm AP and 0.7mm ML; not considered here). Tetrodes were made by twisting and bundling $4 \mathrm{NiCr}$ wires (diameter $13 \mu \mathrm{m}$; Sandvik Palm Coast, Palm Coast, FL), followed by gold electroplating to an impedance of 200-300 kOhm. Electrodes were gradually advanced for 2-3 weeks following surgery to desired depths, after recovery and 
625

626

627

628

629

630

631

632

633

634

635

636

637

638

639

640

641

642

643

644

645

646

647

648

649

650

concurrent with pre-training. The hippocampal formation and advancement into the pyramidal layer was identified by characteristic LFP patterns such as presence of sharp-wave ripples (SWRs), SWR polarity and theta modulation. At the end of the experiment, 24 hours prior to euthanasia, animals were anesthetized (1-2\% isoflurane) and a current (30 $\mu \mathrm{A})$ was passed through each tetrode to form lesions at their tips for localization. Animals were later euthanized (Beuthanasia $200 \mathrm{mg} / \mathrm{kg}$ ) and perfused transcardially with 4\% formaldehyde using approved procedures. Brains were then fixed in $4 \%$ formaldehyde and $30 \%$ sucrose, cut into $50-\mu \mathrm{m}$ sections, stained with cresyl violet, and imaged for verification of lesions indicating tetrode localization.

\section{Electrophysiology and data acquisition}

All tetrodes were referenced with respect to a cerebellar ground screw. For each animal, one tetrode in corpus callosum above the hippocampal layer served as a hippocampal reference electrode. All behavioral and electrophysiological data was acquired using a SpikeGadgets system (SpikeGadgets, San Francisco, CA). Digital electrophysiological data was acquired using 128-channel digitizing headstages, sampled at $30 \mathrm{kHz}$ and saved to disk, with spike data bandpass filtered between $600 \mathrm{~Hz}$ and $6 \mathrm{kHz}$, and local field potential (LFP) bandpass filtered between $0.5 \mathrm{~Hz}$ to $400 \mathrm{~Hz}$ and down sampled to $1.5 \mathrm{kHz}$. Input and output triggers for behavioral and environmental data (eg. reward delivery) were recorded at 1-ms resolution and synchronized to electrophysiological data. Animal movement and behavior was recorded and tracked using an overhead color CCD camera (30 fps), with animal head position, speed, and orientation indicated by color LEDs affixed to the headstage apparatus and microdrive. Cameras were calibrated to provide a resolution of $0.1 \mathrm{~cm} /$ pixel, and spatial extent of LEDs permitted a tracking resolution of $\sim 2 \mathrm{~cm}$.

\section{Unit identification and inclusion}



$40 \mu \mathrm{V}$ in the filtered spike band for hippocampus (CA1). Spikes were then manually sorted into

653 putative units as previously described (Jadhav et al., 2012; Jadhav et al., 2016; Tang et al.,

654 2017; Shin et al., 2019). Briefly, candidate unit spiking had clustering parameters extracted

655 (spike width on each channel, spike amplitude, and principal components), and were clustered 656 using a custom Matlab (MathWorks, Natick, MA; RRID: SCR_001622) cluster visualization

657 program (MatClust). Clusters were judged based on waveform shape, isolation distance, and 658 lack of ISI violation. Only well isolated and stable putative excitatory units were included, with 659 putative interneurons identified and excluded based on average firing rate $\geq 15 \mathrm{~Hz}$ and spike 660 width criteria, as previously described (Jadhav et al., 2016; Tang et al., 2017; Shin et al., 2019).

661 Further, only neurons which fired at least 100 spikes in each session were included for further analysis.

Spatial maps and linearization

Two-dimensional occupancy-normalized spatial firing maps were calculated for each unit smoothed with a 2D Gaussian $(2 \sigma, 6 \mathrm{~cm}$ wide), excluding spiking during high ripple power (>3 SD ripple band power, see LFP collection and high-theta segmentation). The linearized spiking activity of each cell was then computed by first assigning the rat's linear position along the 2D

670 skeleton of the four possible linear behavioral trajectories (center arm reward well to outer arm

671 reward well for outbound trajectories, and the converse for inbound trajectories; (Frank et al., 672 2000; Jadhav et al., 2016)). Spiking and animal occupancy closest to each linear $2 \mathrm{~cm}$ bin on 673 these four trajectories was then used to calculate the smoothed, occupancy-normalized linear

674 firing rate for correct trajectories. A peak firing rate of $3 \mathrm{~Hz}$ or greater was required for a cell to 675 be considered a place cell in CA1 (Jadhav et al., 2016). These linearized trajectories with occupancy-normalized firing rates were used in all subsequent decoding analyses. 
High-theta segmentation and REM state identification

LFP was band pass filtered in the delta (1-4 Hz), theta $(6-12 \mathrm{~Hz})$, and ripple bands (150$250 \mathrm{~Hz}$ ) using zero phase IIR Butterworth filters. We determined envelopes and phases by

681 Hilbert transform, and took the ratio of the theta to delta envelopes at each time point for every 682 hippocampal tetrode. High theta periods during behavior were detected using criteria for theta 683 power, running speed, and exclusion of sharp-wave ripples (SWRs). Specifically, behavioral 684 high theta windows were assigned as uninterrupted time periods at least one theta cycle long $685(\sim 100 \mathrm{~ms})$ when the smoothed $(1 \sigma, 8$ s long) mean theta/delta ratio exceeded 2 , no SWRs were 686 detected with a 3 SD threshold in the absolute power of the ripple band, and animal speed was greater than $3 \mathrm{~cm} / \mathrm{s}$. REM state identification proceeded in the same way, with the exception that animal speed could not exceed a threshold of $3 \mathrm{~cm} / \mathrm{s}$. REM states were identified as previously described (Kay et al., 2016; Tang et al., 2017), with candidate sleep periods identified as times with speed less than a threshold of $3 \mathrm{~cm} / \mathrm{s}$ preceded by extended immobility periods (speed $<3 \mathrm{~cm} / \mathrm{s}$ ) for at least $30 \mathrm{sec}$. REM states were identified as periods within candidate sleep periods with the same conditions for high theta/delta power and low ripple power as behavioral theta periods. LFP and position data for sleep state were also visually inspected for accuracy. Peak theta frequencies per animal and session were determined by computing the power spectrum for theta filtered LFP and taking the maximum peak in the theta range.

Phase locking, phase reversing, and preferred theta phase tetrode located in corpus callosum, as previously described (Jadhav et al., 2016). For a given behavioral session (sleep or wake in a given behavioral epoch), all neurons with >100 spikes in

701 valid theta times were used for computing phase sensitivity metrics (described above as speed 702 during correct trials $>3 \mathrm{~cm} / \mathrm{s}$ and ripple band power $<3 \mathrm{SD}$ ). Phase locking was computed using 
703

704

705

706

707

708

709

710

711

712

713

714

715

716

717

718

719

720

721

722

723

724

725

726

727

a Rayleigh's $z$ test for non-uniformity on the theta phases of the chosen candidate spikes in a given behavioral session. Those neurons which had a Rayleigh's z p value of $<0.05$ were considered to be phase locked in a given behavioral session and used for determining preferred population theta phase preferences. Only neurons which had at least one session in REM and wake were used for segmentation of phase reversing neurons in REM, classified as neurons with preferred mean theta phases centered on $230^{\circ}$ and within $90^{\circ}$ (preferred mean theta phases $\geq 140^{\circ}$ and $\leq 320^{\circ}$ ) similar to (Mizuseki et al., 2011).

\section{Theta cycles and decoding}

Within high theta time windows detected as described above, the troughs $\left(0^{\circ}\right)$ (or peaks $\left(180^{\circ}\right)$, in the case of REM sleep decoding) of the hippocampal theta-filtered LFP were identified and used to segment valid theta cycles, discarding cycles where phase was ambiguous or reset, as previously described (Johnson and Redish; Gupta et al., 2012; Feng et al., 2015; Wu et al., 2017). Due to the properties of CA1 pyramidal cells reversing their preferred phases in sleep (Mizuseki et al., 2011), REM theta sequences were segmented using a peak-topeak method. Only theta cycles with at least 3 simultaneously active template cells were analyzed, and theta cycle decoding of place was implemented as previously described (Johnson and Redish; Gupta et al., 2012; Wu et al., 2017). A Bayesian decoder was used to calculate the probability of the animal's location given the firing rate templates of the neurons that fired and their spikes that occurred in each time window, where time windows were sliding 20ms temporal bins with $5 \mathrm{~ms}$ of overlap. (Zhang et al., 1998; Davidson et al., 2009; Karlsson and Frank, 2009; Gupta et al., 2012; Pfeiffer and Foster, 2013). Briefly, the probability of the animal's position (pos) across all total spatial bins $(S)$ given a time window $(t)$, containing Poisson spiking (spikes) of independent units is 


$$
P(\text { pos } \mid \text { spikes })=\frac{P(\text { spikes } \mid \text { pos }) P(\text { pos })}{P(\text { spikes })}
$$

Normalizing over $P$ (spikes) and using a uniform prior $P(p o s)$ to avoid spatial decoding bias, we

731 get

$$
\mathrm{P}(\text { pos } \mid \text { spikes })=\frac{U}{\sum_{j=1}^{S} U} \quad \text { where } \quad U=\left(\prod_{i=1}^{N} f_{i}(\text { pos })^{n_{i}}\right) e^{-t \sum_{i=1}^{N} f_{i}(\text { pos })}
$$

Where $f_{i}$ (pos) is the occupancy normalized 1D firing rate map for the $i$-th unit, $N$ is the total number of active units, $n_{i}$ is the number of spikes fired by a particular $i$-th unit, and $t$ is a time window (in this case, the entire theta cycle). This was computed using the firing rate template the position with the maximum probability in that time bin.

\section{Theta Sequence Validation: Population Vector Overlap}

744 In order to confidently differentiate decodes between different running directions in both wake

745 and REM sleep, the similarity of the place-cell population between every behavioral trajectory in

746 wake was computed using the population vector overlap (PVO) technique (Ravassard et al.,

747 2013). The population vector (PV) was the group vector of all place cells in a certain linear

748 position bin in a given epoch. The PVO was then defined as the vector dot product between the

749 PVs across all linear positions in every epoch and every trajectory combination:

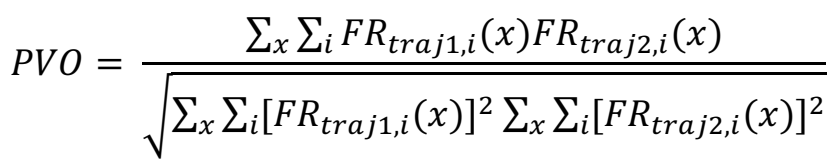


751 Where $\mathrm{FR}_{\mathrm{traj} 1, \mathrm{i}}(\mathrm{x})$ is the firing rate of the $\mathrm{i}$-th place cell at the linear position $\mathrm{x}$ along the track on

752 behavioral trajectory traj1, and $\mathrm{FR}_{\text {traj2, }}(\mathrm{x})$ is the firing rate of the $\mathrm{i}$-th place cell at the linear

753 position $x$ along the on behavioral trajectory traj2. The PVO is a singular metric from 0 to 1 , with

7541 representing identical population place-field templates. To determine the significance of

755 PVOs, we created a shuffled surrogate that permuted trial to trial assignments of spiking to

756 behavioral trajectories for every epoch (behavior epochs 1 and 2 were combined due to low

757 number of trials in epoch 1), repeating this procedure 1000 times and recomputing a null

758 distribution to test our actual PVO values against. Data for sessions 1-2 for one animal was

759 omitted from sequence analyses due to lack of sufficient number of trials.

760

761

Theta Sequence Validation: Sequence Scores

762

For every putative theta sequence and every trajectory decoding template, the slope/line

763

fitting procedure from (Davidson et al., 2009; Kloosterman et al., 2014; Feng et al., 2015) was

764

used. Briefly, this decoding technique produces a line representing a putative theta sequence

765

with a given slope $(V)$ and intercept $(\rho)$ maximizing the amount of probability captured within a

766

20-cm area around a line/theta sequence, fitted to the decoded posterior:

767

768

$$
R(V, \rho)=\frac{1}{n} \sum_{k=0}^{n=1} P_{r}(|p o s-(\rho+V * k * \Delta t)| \leq d)
$$

Maximizing the value $R$, where $\Delta t$ is the moving window step $(5 \mathrm{~ms}), \mathrm{d}$ is $10 \mathrm{~cm}( \pm 10 \mathrm{~cm}$

vicinity), theta time bins $(k)$, and total time bin number $(n)$. For time bins where a fitted line

772 and intercept parameters were densely sampled to determine the values that maximized this

773 sequence score.

775 Theta Sequence Validation: Shuffles 

posterior containing a theta sequence, a null distribution was bootstrapped by randomly

778

permuting this posterior 1000 times and computing the normalized sequence score as described above. Two permutation tests were carried out to construct two null distributions randomly permuting the order of posterior columns and thus permuting time bins (henceforth known as time shuffle), and randomly circularly shifting columns and thus permuting space to which the posterior decoded (henceforth known as space shuffle).

\section{Theta Sequence Selection}

After computing sequence scores for both null distribution shuffles and the true sequence score, the following was evaluated in order to select theta sequences from noisy or non-existent theta sequences. In practice, this selection required the following heuristics that imposed strict criteria to minimize false positives in theta sequence identification, at the risk of possible false negatives. A "true theta sequence" sequence score had to exceed the $99^{\text {th }}$ percentile of both space and time shuffled distributions. No more than $30 \%$ of the track could be traversed from one posterior time bin to another in the "decoded position" of the posterior, in order to enforce contiguity, used previously as a metric deemed the decode jump distance (Silva et al., 2015). No more than 3 bins (15 ms) of contiguous decoding ambiguity in the prior (time bins where no spikes occurred, or time bins where the posterior was uniform, and no maximally decoded spatial bin could be ascertained). This cutoff was used to enforce contiguity of decoding, rather than contiguity of decoded space. In addition, the virtual speed of the decode was restricted to the range of $1 \mathrm{~m} / \mathrm{s}$ to $10 \mathrm{~m} / \mathrm{s}$, to avoid any possibility of stationary decodes, which have been described previously (Farooq and Dragoi, 2019; Muessig et al., 2019) but were beyond the scope of this analysis, as well as decodes that traversed the track faster than a single theta cycle, which were deemed to be artifactual or ripple-like given previous literature. In addition, only the tail ends of all sequence scores were considered, discarding any 
802

803

804

805

806

807

808

809

810

811

812

813

814

815

816

817

818

819

820

821

822

823

824

825

826

827

sequences whose true sequence scores were below the median of the population. With these cutoff criteria, what remains is determining which of the four decoding templates (OL, IL, OR, IR) is the correct decoding template. In practice, this could be determined by using the true trajectory the animal was currently on for awake theta sequences, but not during REM sequences. To ameliorate this, after these thresholding procedures, the decoding template that had the maximum true sequence score was chosen, similarly for both awake and REM theta sequences.

\section{Average theta sequences}

Average theta sequences were constructed as follows. Each posterior of a selected theta sequence during awake behavior was shifted such that the position of the rat was at $0 \mathrm{~cm}$, with theta sequence decodes for both decoding directions merged. The first $30 \mathrm{~cm}$ behind and ahead of an animal at a given theta sequence decode were then shown. For REM theta sequences, a similar procedure was carried out with some exceptions. Sleep decodes were aligned such that the theta cycle time midpoint was considered, and the decoded position at that timepoint of the sequence posterior was used to center the decode to $0 \mathrm{~cm}$. Due to the nature of the slope-fitting maximization parameters, negative slopes were not restricted, that is, we allowed for theta sequences with negative slopes to be found in this maximization procedure. In practice, this means that we allowed for the possibility of a theta sequence to occur against the animal's direction of travel for a given trajectory, i.e. reverse theta sequences.

\section{Average theta sequences- quadrant scores and validation}

To quantify the structure of average theta sequences over time periods, we computed the quadrant ratio for each given posterior (Farooq and Dragoi, 2019). Briefly, the quadrant ratio gives a value from -1 to 1 indicating how much probability within the decoded posterior exhibits a canonical theta sequence, where the posterior is split into four quadrants, centered on the 
midpoint of the theta sequence on the $x$ axis and centered along a relative position on the $y$ axis. Quadrants II and III are located early in the theta cycle, while quadrants I and IV are located late in the theta cycle; while quadrants I and II are oriented in front of a spatial midpoint, and quadrants III and IV are located behind a spatial midpoint.

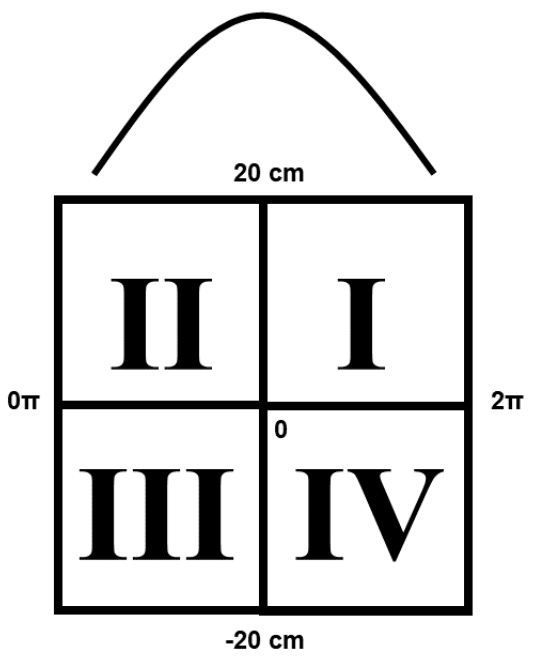

832

A canonical theta decode posterior exhibits the following structure, where a decode sweeps from behind the animal spatially early in a theta sequence (Quadrant III/QIII) to spatially in front of the animal late in a theta sequence (Quadrant I/QI) over time. Summing the total probability of the posterior in a given window that falls into QIII/QI, subtracting QII/QIV and normalizing by total probability in this window would lead to quadrant score values closer to 1 , indicating a stronger likelihood of a structured forward theta sequence. A reversed theta sequence would then be ahead of the animal's current position early in a theta sequence (Quadrant II/QII) and behind the animal's current position late in a theta sequence (Quadrant IV/QIV), where values closer to -1 would indicate a strong likelihood of a structured backward theta sequence. 
Quadrant Ratio $=\frac{\sum\left(q_{1}, q_{3}\right)-\sum\left(q_{2}, q_{4}\right)}{\sum\left(q_{1}, q_{2}, q_{3}, q_{4}\right)}$

Each theta decode posterior at least $20 \mathrm{~cm}$ from either end of the track was centered either according to the animal's current spatial position (in wake), or midpoint of the putative theta decode (in REM), and then truncated to a local spatial area $\pm 20 \mathrm{~cm}$ from this spatial midpoint for further quadrant score computation. Similar to validation of theta sequence decodes, the following procedure was carried out to create null distributions to test for significance. Each

851 theta sequence posterior used for quadrant ratio quantification was randomly permuted 500

852 times, again permuting column order (time shuffle, permuting theta time bins) or circularly shifted along columns (space shuffle, permuting the spatial structure of the posterior per time bin), and quadrant ratios were recomputed. The real quadrant score for a given theta decode posterior was then compared to its corresponding null distribution in either shuffle type, and its percentile plotted.

Since the line fitting procedure for quantification of theta sequences returns an assumed contiguous decode, we can also visualize the starts and ends of theta sequences, their spatial extents, and the distributions of decodes along the length of the tracks over time. To do this, we constructed quiver plots, as in (Wu et al., 2017), with the following properties. For a given theta sequence, an arrow is plotted where the $x$-axis indicates decoded positions- circles at the ends of arrows represent theta sequence decode start positions, and triangle ends represent theta sequence decode end positions. The $y$-axis position of the arrow delineates the ordinal value of the theta sequence, ordered according to decode start location on the track. Track arm extents

867 are skeletonized with vertical lines indicating maze corners. To summarize the spatial extent of 868 theta sequences, sequence extents are binned in 1-cm bins, then plotted as histograms of total decode extents, showing total spatial coverage of decodes, as well as any biases or over 
870

871

872

873

874

875

876

877

878

879

880

881

882

883

884

885

886

887

888

889

890

891

892

893

894

representation of space. Decodes with 2 or more phase-shifting cells, which comprise of at least $25 \%$ of all participating place cells which shift their phase in REM sleep, are considered to be sequences which had an ensemble of shifting cells. Qualitatively similar results were obtained using a $50 \%$ threshold.

To quantify the spatial coverage decodes in REM and wake by phase-shifting ensembles and non-shifting ensembles, we computed the coverage ratio between the two:

$$
\text { coverage ratio }=\frac{\sum \text { bins }_{\text {shifter }}-\sum \text { bins }_{n o n-s h i f t e r}}{\left(\sum \text { bins }_{\text {shifter }}+\sum \text { bins }_{n o n-s h i f t e r}\right)}
$$

For each behavioral epoch, we subtracted the summed coverage in non-shifting ensembles from the coverage in shifting ensembles, normalized by the total number of covered bins in both ensembles, which resulted in a number varying from -1 (track over-representation by nonshifting ensembles) to 1 (higher track representation by shifting ensembles).

\section{Data Visualization}

Colormaps used throughout were modified from the matplotlib package and adjusted with vscim, with the goal of being perceptually uniform (Thyng et al., 2016). Figure layout was generated in part using a ggplot2-like package created for MATLAB, Gramm (Morel, 2018).

\section{Data Availability and Interactive dataset visualization}

The entire dataset of putative theta sequences for wake and REM is available for visualization at www.github.com/JadhavLab/ThetaSequencesInREM/ . To visualize this dataset of putative theta sequences, we used an unsupervised non-linear dimensionality reduction technique known as Uniform Manifold Approximation and Projection (UMAP) (Mclnnes et al., 2018). Briefly, UMAP is a manifold learning technique that finds a lower dimensional embedding of higher dimensional data, much like t-SNE, though UMAP preserves both global and local 
895

896

897

898

899

900

901

902

903

904

905

906

907

908

909

910

911

912

913

914

915

916

917

918

919

920

structure (see https://umap-learn.readthedocs.io/en/latest/ for details). Each point indicates a single putative theta sequence prior to our selection criteria, color coded according to line-fitting score (warmer colors indicating lower line-fitting score, while cooler colors indicating higher linefitting score, see colorbar for values). Each point, when hovered over, shows a decoded sequence, with $\mathrm{x}$ axis indicating time, $\mathrm{y}$ axis indicating track space, and color indicating probability of decode in that time $x$ space bin (saturated for visualization). Drop down menus toggle between line-fitting score and selected/non-selected sequence color maps (selected decodes in dark blue, and non-selected decodes in yellow), respectively. An optional alpha/opacity dropdown can be used to indicate/highlight selected/non-selected decodes (to highlight selected decodes, choose the options, Values = selected; Alpha = selected_alpha).

(1)

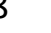

(1)

0

1

2


921

922

923

924

925

926

927

928

929

930

931

932

933

934

935

936

937

938

939

940

941

942

943

944

945

946

\section{FIGURE LEGENDS}

\section{Figure 1- REM sleep and distinct behavioral trajectory representations in CA1}

(A) Schematic of one behavioral epoch; a W track session (Run/Wake) and subsequent sleep session (Sleep/REM). A successful behavioral sequence in the W track spatial alternation task requires alternation between outbound arm visits (red wells) and returns to the center arm (blue wells) for a total of four trajectories depicted from left to right: Outbound Left (OL), Inbound Left (IL), Outbound Right (OR), and Inbound Right (IR).

(B) Illustration of the single day $\mathrm{W}$ track acquisition task, consisting of eight behavioral epochs (one run session, followed by one sleep session) over the course of one day. An initial sleep session prior to the first track exposure was not used.

(C) (Left) Schematic of all hippocampal tetrode locations in all animals, one color per animal ( $n=6$ animals). (Right) Coronal brain section showing locations of two CA1 tetrode lesions (red arrows).

(D) Segmentation of SWS and REM during sleep sessions. (i) shows the theta delta ratio for a given sleep session, with theta/delta ratio greater than 2 highlighted in black. Animal speed in (ii) is consistently immobile or below a threshold of $3 \mathrm{~cm} / \mathrm{s}$ for all REM sessions. Ripple power is consistently low in REM sleep (iii), with no ripple events exceeding 3sd. Note the change in overall spike rate and place cell activity in (iv).

(E) Sleep fraction of REM (blue) does not change over behavioral epochs (all $p>0.81$, KruskalWallis test with Tukey-Kramer correction for multiple comparisons).

(F) Animal performance in wake sessions (orange), calculated as the total number of correct inbound and outbound trials over the total number of trials. Animal performance asymptotes behavioral epoch \#5, defined as the first behavioral epoch where performance is significantly 
947

948

949

950

951

952

953

954

955

956

957

958

959

960

961

962

963

964

965

966

967

968

969

970

971

different from the first behavioral epoch $(p<0.01$ for behavioral epoch comparison 1 vs. 5 , Kruskal-Wallis test with Tukey-Kramer correction for multiple comparisons).

(G) Firing rate curves of all place cells for one animal in one behavioral epoch along a linearized trajectory $(0-200 \mathrm{~cm}$, normalized to peak rates). From left to right, cells are sorted according to peak place field locations on that trajectory.

(H) Population vector overlap (PVO) correlations of place cells between each trajectory for every behavioral epoch (light), and the corresponding $95 \%$ Cls of trial permuted shuffles (dark regions shows shuffles for all trajectory comparisons). All trajectory templates are distinct from one another throughout the experiment (all $p<0.01$, permutation tests). Epochs 1 and 2 were combined due to low number of trials in the first epoch during permutation. Outbound Left (OL), Inbound Left (IL), Outbound Right (OR), and Inbound Right (IR).

Data are means (solid lines) \pm SEM (shaded area). ${ }^{*} P<0.05$; ns, not significant.

\section{Figure 2- Theta phase preference in wake and REM}

(A) Distribution of mean preferred theta phases of all place cells that showed significant thetaphase modulation ( $p<0.05$, Rayleigh's z-test) in wake (orange) and REM sleep (blue). The two distributions of preferred phases differ in run and REM sleep ( $p<0.005$, Kuiper's test).

(B) Distribution of mean preferred theta phases for both run and REM sleep. Only neurons which had at least one epoch of significant theta phase modulation in both run and REM are shown. Neurons with $<=140^{\circ}$ or $>=320^{\circ}$ preferred phases centered around peak preferred phase during REM sleep are designated as shifting cells (red), while the remainder are considered non shifting cells (black), similar to (Mizuseki, 2011).

(C) Example individual phase shifting cell examples, with histograms of spike theta phases in wake (orange) and REM sleep (blue). 
972 (D) Distribution of preferred theta phases of all place cells that showed significant theta-phase

973 modulation per behavioral epoch, showing both shifting (red) and non-shifting (black) cells. Data

974 are circular means (solid lines) \pm bootstrapped 95\% Cls (shaded area). Note that the circular Y-

975 axis wraps around at 0 and 360 .

976 (E) Same as in C, split into wake (left) and REM sleep (right) epochs. (Left) Preferred phase of

977 shifting and non-shifting cells during wake is similar for all epochs ( $p=0.52$, Watson-Willams

978 test). (Right) Preferred phase of shifting and non-shifting cells is different over all epochs in

$979 \operatorname{REM}(p<1 \mathrm{e}-99$, Watson-Willams test).

980 Data are circular means (solid lines) \pm bootstrapped $95 \%$ Cls (shaded area). ${ }^{*}<0.05$; ns, not 981 significant.

982 (F) Same as in E, summarized across all epochs as polar plots, split into wake (left) and REM 983 sleep (right) sessions.

984 (G) Mean theta spectra in wake and REM for all animals.

985 (H) Mean theta frequencies for all animals do not differ in wake vs. REM sleep $(p=0.4080$, 986 Friedman Test).

987 Data in (G)-(H) are means (solid lines) \pm SEM (shaded area). ${ }^{*} P<0.05$; ns, not significant.

Figure 3- Theta sequence decoding and significance in wake and REM

(A-F) Example decoded theta sequences in wake (A-C) and REM (D-F).

(A) Example decoded theta sequence in wake. This wake sequence significantly decodes an outbound right trajectory in the forward direction. (Top Left) The raw LFP trace of this theta cycle (black), and corresponding theta filtered LFP (rainbow indicating phase). (Bottom Left)

995 Spikes and neurons in this theta sequence, sorted by peak firing of the chosen trajectory.

996 (Bottom Right) Firing rate curves per trajectory type for the neurons at the bottom left, sorted 997 and normalized according to peak firing of the chosen trajectory, highlighted in red. (Top Right) 
Decoded posteriors for each trajectory type, with warmer colors indicating higher spatial bin probability and corresponding putative theta sequence line fits overlaid. Arrows to the right

1000 indicate the animals' direction of travel in this trajectory type, in order to differentiate forward and

1001 reverse decodes. Titles indicate normalized line fitting scores. Chosen trajectory and

1002 contributing firing rate curves highlighted in red, with direction of travel arrows in orange.

1003 (B) Same as previous. This wake sequence significantly decodes an outbound left trajectory in 1004 the reverse direction.

1005 (C) Same as previous. This wake sequence significantly decodes an outbound left trajectory in 1006 the forward direction.

1007 (D) Example decoded theta sequence in REM. This REM sequence significantly decodes an 1008 inbound left trajectory in the forward direction.

1009 (E) Same as previous. This REM sequence significantly decodes an outbound left trajectory in 1010 the forward direction.

1011 (F) Same as previous. This REM sequence significantly decodes an outbound right trajectory in 1012 the reverse direction. The inbound left trajectory was not chosen due to violation of the jump 1013 distance criterion.

1014 (G) Proportion of significant theta sequences over behavioral epochs for wake (orange) and 1015 REM sleep (blue). Wake theta sequences consistently had a higher proportion of significant 1016 decodes than REM sleep ( $p=3.48 e-07$, Friedman Test).

1017 (H) Proportion of forward theta sequences over behavioral epochs for wake (orange) and REM 1018 sleep (blue). Wake theta sequences consistently had a higher proportion of forward decodes 1019 than REM sleep ( $p=1.49 \mathrm{e}-06$, Friedman Test)

1020 (I) Mean absolute theta decode slopes over behavioral epochs. REM theta sequences 1021 consistently had a higher decode slope $(p=2.29 e-10$, Friedman Test). 
(J) Mean absolute theta decode extents over behavioral epochs. Similar to mean absolute

1023

1024

1025

1026

1027

1028

1029

1030

1031

1032

1033

1034

1035

1036

1037

1038

1039

1040

1041

1042

1043

1044

1045

1046

1047

decode slopes, REM theta sequences consistently had a higher decode extent $(p=2.29 \mathrm{e}-10$, Friedman Test).

Data in (G)-(J) are means (solid lines) \pm SEM (shaded area). ${ }^{\star} P<0.05$; ns, not significant.

\section{Figure 4- Theta sequence propagation in the forward and reverse direction}

(A) Average theta sequences with respect to animal position in the forward (top row) and reverse direction (bottom row) during run sessions over behavior. Heat indicates maximum decoded probability over theta decode bins and relative distance from the animal position.

Overlaid horizontal line indicates the decode midpoint, with overlaid mean LFP theta waveform and waveform SEM as shaded area.

(B) Permutation test results for all forward theta sequences during wake, centered on animal position. Permutation tests were carried out using quadrant scores of individual decode posteriors versus the corresponding quadrant scores of randomly permuted decode posteriors, in order to create quadrant score null distributions. Lighter and darker boxplots indicate distributions of $p$-values using two different permutation strategies, shuffling decode posteriors in both space (circular permutation of space in each decode time bin) and time (permutation of decode posterior columns), respectively. Median p-values of all decodes over all epochs and tests are lower than 0.05 (thick dashed horizontal line).
(C) Same as in
$\mathbf{B}$, for reverse (quadrant score less than 0 )
0) theta sequences during wake, centered on animal position. Median p-values are consistently higher than 0.05 , indicating significant reverse decodes are rare relative to permuted decodes as measured by quadrant score.

(D) Same as in A but with respect to decode midpoint in the forward and reverse direction during REM sleep sessions over behavior. 
1048 (E) Same as in B, for forward theta sequences during REM sleep, centered on decode midpoint. 1049 Median p-values are consistently lower than 0.05 , indicating significant forward decodes relative 1050 to permuted decodes as measured by quadrant score.

1051 (F) Same as in C, for reverse theta sequences during REM sleep, centered on decode midpoint. 1052 Median p-values are consistently lower than 0.05, indicating significant reverse decodes in REM 1053 sleep relative to permuted decodes as measured by quadrant score.

Figure 5- Theta sequence content and participation of phase-reversing cells

1057 (A) Relative proportions of decodes comprising phase-shifting vs. non phase-shifting cells in wake (orange) and REM sleep (blue) over behavioral epochs. Decodes with 2 or more phaseshifting cells, which comprise of at least $25 \%$ of all participating place cells which shift their phase in REM sleep, are considered to be "shifter sequences" comprising shifting cells. Values greater than 0 indicate decodes with less phase shifting cells (or, more non-shifting cells); values near 0 indicate a balance between decodes with and without shifting neurons, and values below zero indicate decodes with more shifting neurons. Note the statistically significant difference between the proportion of wake and REM shifter and non-shifter sequences $(p=$ 0.0043, Friedman Test).

(B) Theta sequence trajectories for wake (Top) and REM (Bottom) sessions in the (left) forward 1067 and (right) reverse decode directions for an early learning session in one animal, sorted 1068 according to decode start position. Only forward sequences are shown in wake. Circles on the $1069 x$-axis indicate decode starts, arrowheads on the x-axis indicate direction of decode ends. Lines 1070 in red indicate decodes with more than 25\% reversing cells (termed "phase-reversing or shifter 1071 cell sequences"), while lines in black indicate less than $25 \%$ phase reversing cells. Top 1072 histograms indicate decode coverage of the track, split into phase shifting and non- phase 
1073 shifting theta sequences. For REM sleep epoch, note dearth of phase reversing cell (red)

1074 sequences.

1075 (C) Theta sequence trajectories for wake (Top) and REM (Bottom) sessions in the (left) forward 1076 and (right) reverse decode directions for a late performance session. Only forward sequences 1077 are shown in wake. Note that REM sleep epoch has similar proportions of forward and reverse 1078 sequences and included many phase reversing cell (red) sequences. Note also increase in 1079 spatial coverage for phase reversing cell sequences in REM (red histogram in top row) for this 1080 late performance session as compared to early learning session in $\mathbf{A}$.

1081 (D) Coverage histograms of all epochs, and behavioral states, with shifting sequence decodes 1082 in red and non-shifting sequence decodes in black. In REM, sequence decodes containing 1083 phase shifting neurons emerge over learning (note epochs 5-8), corresponding with asymptotic 1084 behavioral performance in run sessions.

1085 (E) Quantification of sequence decode coverage by shifter vs. non-shifter sequences over 1086 epochs for wake (orange) and REM (blue). Overall, there was a statistically significant

1087 difference in the normalized bin decode proportion for REM over epochs (blue, $p=0.0016$, 1088 Kruskal-Wallis test). Wake sequence decodes (orange) with phase shifting cells do not show 1089 this effect over epochs $(p=0.0726$, Kruskal-Wallis test). 


\section{SUPPLEMENTARY FIGURE LEGENDS}

Figure S1- Theta sequences and their properties, Related to Figure 3

1103 (A) 0.5 seconds of theta decodes during waking behavior. (i) Theta filtered LFP, scaled to

1104 maximum of the entire session, theta phase in (rainbow). Note parsing of cycles from trough to

1105 trough during wake. (ii) Spikes from all neurons, sorted according to peak field position along

1106 the track (y-axis, 0-200cm). Animal's current position is shown by the orange line. (iii)-(vi) theta

1107 cycle decodes from trajectory templates ordered vertically as OL, IL, OR, IR respectively.

1108 Heatmap indicates probability, with the x-axis indicating decode time-bin, and the y-axis

1109 indicating decoded position $(0-200 \mathrm{~cm})$, with the same position scale as (ii). Line fits of putative

1110 theta sequences shown with dotted arrowhead lines (significantly decoded theta sequence

1111 shown as solid red line with arrowhead). Animal's current position in orange, as in ii.

1112 (B) Same as A, 0.5 seconds of theta decodes during REM sleep. Note parsing of theta cycles

1113 from peak to peak (i), and the lack of the animal's current position (ii).

1114 (C) Normalized line fitting scores, calculated as the summed captured probability of the putative

1115 theta sequence in each decode time bin divided by the summed theoretical maximal captured

1116 probability in each time bin (wake and REM sleep distributions are different, $p<1$ e-99, rank-sum

1117 test).

1118 (D) Absolute virtual decode velocities of putative theta sequences. Only theta sequences with

1119 virtual velocities greater than $1 \mathrm{~m} / \mathrm{s}$ or less than $10 \mathrm{~m} / \mathrm{s}$ were included for further analysis (wake

1120 and REM sleep distributions are different, $p<3.41 \mathrm{e}-59$, rank-sum test).

1121 (E) Absolute decoding distance of all putative theta sequences in wake (orange) and REM sleep

1122 (blue), calculated as the absolute distance from the first decoded spatial bin to the final decoded

1123 spatial bin (wake and REM sleep distributions are different, $p<1.46 e-47$, rank-sum test).

1124 (F) Time bin shuffle percentile. For a single given posterior representing a putative theta 
1125

1126

1127

1128

1129

1130

1131

1132

1133

1134

1135

1136

1137

1138

1139

1140

1141

1142

1143

1144

1145

1146

1147 Figure S2- Additional theta sequence examples; Related to Figure 3

1148 Same as Figure 3

1149 (A-E) Examples of wake theta sequences

1150 (F-J) Examples of REM theta sequences the percentile of the true normalized line fitting score in comparison to the distribution of the shuffled line fitting scores. Only theta sequences which exceeded the $99^{\text {th }}$ percentile were included for further analysis (wake and REM sleep distributions are different, $p<7.96$ e-54, rank-sum test).

(G) Space shuffle percentile. For a single given posterior representing a putative theta sequence, posterior columns (representing decoded space) were circularly permuted 1000 exceeded the $99^{\text {th }}$ percentile were included for further analysis (wake and REM sleep distributions are different, $p<3.28$ e-33, rank-sum test).

(H) Maximum normalized track jump distance, computed as the maximum track distance a putative decode travelled from one time bin to another. Only theta sequences with jump distributions are different, $\mathrm{p}<8.32 \mathrm{e}-20$, rank-sum test).

(I) Mean absolute theta decode slopes over behavioral epochs per individual animal.

(J) Mean absolute theta decode extents over behavioral epochs per individual animal.

Data in (I)-(J) are means (points) $\pm 95 \% \mathrm{Cl}$ (lines).

sequence, time bins (columns) were permuted 1000 times at random, and normalized line fitting scores were calculated for each of these shuffled posteriors. The time bin shuffle percentile is times at random, and normalized line fitting scores were calculated for each of these shuffled posteriors. The space shuffle percentile is the percentile of the true normalized line fitting score in comparison to the distribution of the shuffled line fitting scores. Only theta sequences which distances less than $30 \%$ of the track were included for further analysis (wake and REM sleep 


\section{Figure S3- Theta asymmetry; Related to Figure 4}

1154 (A) Mean theta waveform asymmetries in wake are not significantly different over epochs

$1155(p=0.1054$, Kruskal-Wallis test $)$, with theta asymmetries in $(\mathbf{C})$ shown as mean asymmetry score 1156 and $\mathrm{Cl}$ per animal.

1157 (B) Mean theta waveform asymmetries in REM sleep are not significantly different over epochs $1158 \quad(p=0.609$, Kruskal-Wallis test $)$, with (D) same as (C).

1159 Data in (A)-(B) are means (solid lines) \pm SEM (shaded area). ${ }^{*} P<0.05$; ns, not significant. Data 1160 in (C)-(D) are means (points) $\pm 95 \% \mathrm{Cl}$ (lines).

Figure S4- Wake quadrant score quantification according to midpoint alignment; Related to Figure 4

(A) Average theta sequences with respect to decode midpoint in the forward (top row) and reverse direction (bottom row) during run sessions over behavior. Heat indicates maximum

1167 decode probability over theta decode bins and relative distance from the animal. Overlaid 1168 horizontal line indicates the animal position, with mean LFP waveform and waveform SEM as 1169 shaded area.

1170 (B) Permutation test results, for forward theta sequences centered on decode midpoint during 1171 wake. Median p-values are consistently lower than 0.05 , indicating significant forward decodes 1172 relative to permuted decodes as measured by quadrant score.

1173 (C) Same as in B, for reverse theta sequences centered on decode midpoint during wake.

1174 Median p-values are consistently lower than 0.05 indicating significant reverse decodes relative 1175 to permuted decodes as measured by quadrant scores. 


\section{REFERENCES}

1180

1181

1182

1183

1184

1185

1186

1187

1188

1189

1190

1191

1192

1193

1194

1195

1196

1197

1198

1199

1200

1201

1202

1203

1204

1205

1206

1207

1208

1209

1210

1211

1212

1213

1214

1215

1216

1217

1218

1219

1220

1221

1222

1223

1224

\section{REFERENCES}

Alberini CM (2005) Mechanisms of memory stabilization: are consolidation and reconsolidation similar or distinct processes? Trends in Neurosciences 28:51-56.

Bi GQ, Poo MM (1998) Synaptic modifications in cultured hippocampal neurons: dependence on spike timing, synaptic strength, and postsynaptic cell type. Journal of Neuroscience 18:10464-10472.

Buzsáki G (1989) Two-stage model of memory trace formation: a role for "noisy" brain states. Neuroscience 31:551-570.

Buzsáki G (2002) Theta Oscillations in the Hippocampus. Neuron 33:325-340.

Cei A, Girardeau G, Drieu C, Kanbi KE, Zugaro M (2014) Reversed theta sequences of hippocampal cell assemblies during backward travel. Nature Neuroscience 17:719-724.

Danielson NB, Zaremba JD, Kaifosh P, Bowler J, Ladow M, Losonczy A (2016) Sublayer-Specific Coding Dynamics during Spatial Navigation and Learning in Hippocampal Area CA1. Neuron 91:652-665.

Davidson TJ, Kloosterman F, Wilson MA (2009) Hippocampal replay of extended experience. Neuron 63:497-507.

Dragoi G, Buzsáki G (2006) Temporal encoding of place sequences by hippocampal cell assemblies. Neuron 50:145-157.

Drieu C, Zugaro M (2019) Hippocampal Sequences During Exploration: Mechanisms and Functions. Frontiers in Cellular Neuroscience 13:232.

Drieu C, Todorova R, Zugaro M (2018) Nested sequences of hippocampal assemblies during behavior support subsequent sleep replay. Science 362:675-679.

Dudai Y (2004) The neurobiology of consolidations, or, how stable is the engram? Annu Rev Psychol 55:51-86.:51-86.

Dupret D, O'Neill J, Pleydell-Bouverie B, Csicsvari J (2010) The reorganization and reactivation of hippocampal maps predict spatial memory performance. Nat Neurosci 13:995-1002.

Eichenbaum H, Cohen NJ (2004) From Conditioning to Conscious Recollection: Memory Systems of the Brain: Oxford University Press.

Eichenbaum H, Dudchenko P, Wood E, Shapiro M, Tanila H (1999) The hippocampus, memory, and place cells: is it spatial memory or a memory space? Neuron 23:209-226.

Farooq U, Dragoi G (2019) Emergence of preconfigured and plastic time-compressed sequences in early postnatal development. Science 363:168-173.

Feng T, Silva D, Foster DJ (2015) Dissociation between the experience-dependent development of hippocampal theta sequences and single-trial phase precession. J Neurosci 35:4890-4902.

Foster DJ (2017) Replay comes of age. Annu Rev Neurosci 40:581-602.

Foster DJ, Wilson MA (2006) Reverse replay of behavioural sequences in hippocampal place cells during the awake state. Nature 440:680-683.

Foster DJ, Wilson MA (2007) Hippocampal theta sequences. Hippocampus 17:1093-1099.

Frank LM, Brown EN, Wilson M (2000) Trajectory encoding in the hippocampus and entorhinal cortex. Neuron 27:169-178.

Frank LM, Stanley GB, Brown EN (2004) Hippocampal plasticity across multiple days of exposure to novel environments. J Neurosci 24:7681-7689.

Geiller T, Royer S, Choi J-S (2017) Segregated Cell Populations Enable Distinct Parallel Encoding within the Radial Axis of the CA1 Pyramidal Layer. Experimental Neurobiology 26:1-10. 
Genzel L, Spoormaker VI, Konrad BN, Dresler M (2015) The role of rapid eye movement sleep for amygdala-related memory processing. Neurobiol Learn Mem 122:110-121.

Griffin AL, Asaka Y, Darling RD, Berry SD (2004) Theta-Contingent Trial Presentation Accelerates Learning Rate and Enhances Hippocampal Plasticity During Trace Eyeblink Conditioning. Behavioral Neuroscience 118:403-411.

Grosmark AD, Mizuseki K, Pastalkova E, Diba K, Buzsáki G (2012) REM sleep reorganizes hippocampal excitability. Neuron 75:1001-1007.

Gupta AS, van der Meer MA, Touretzky DS, Redish AD (2012) Segmentation of spatial experience by hippocampal theta sequences. Nat Neurosci 15:1032-1039.

Harris KD, Henze DA, Hirase H, Leinekugel X, Dragoi G, Czurko A, Buzsaki G (2002) Spike train dynamics predicts theta-related phase precession in hippocampal pyramidal cells. Nature 417:738-741.

Hölscher C, Anwyl R, Rowan MJ (1997) Stimulation on the positive phase of hippocampal theta rhythm induces long-term potentiation that can Be depotentiated by stimulation on the negative phase in area CA1 in vivo. The Journal of neuroscience : the official journal of the Society for Neuroscience 17:6470-6477.

Huerta PT, Lisman JE (1993) Heightened synaptic plasticity of hippocampal CA1 neurons during a cholinergically induced rhythmic state. Nature 364:723--725.

Huerta PT, Lisman JE (1995) Bidirectional synaptic plasticity induced by a single burst during cholinergic theta oscillation in CA1 in vitro. Neuron 15:1053--1063.

Hyman JM, Wyble BP, Goyal V, Rossi CA, Hasselmo ME (2003) Stimulation in hippocampal region CA1 in behaving rats yields long-term potentiation when delivered to the peak of theta and long-term depression when delivered to the trough. J Neurosci 23:11725--11731.

Izawa S, Chowdhury S, Miyazaki T, Mukai Y, Ono D, Inoue R, Ohmura Y, Mizoguchi H, Kimura K, Yoshioka $M$, Terao A, Kilduff TS, Yamanaka A (2019) REM sleep-active $\mathrm{MCH}$ neurons are involved in forgetting hippocampus-dependent memories. Science 365:1308-1313.

Jackson J, Amilhon B, Goutagny R, Bott J-B, Manseau F, Kortleven C, Bressler SL, Williams S (2014) Reversal of theta rhythm flow through intact hippocampal circuits. Nature neuroscience 17:1362-1370.

Jadhav SP, Kemere C, German PW, Frank LM (2012) Awake hippocampal sharp-wave ripples support spatial memory. Science 336:1454-1458.

Jadhav SP, Rothschild G, Roumis DK, Frank LM (2016) Coordinated excitation and inhibition of prefrontal ensembles during awake hippocampal sharp-wave ripple events. Neuron 90:113-127.

Johnson A, Redish AD (2007) Neural ensembles in CA3 transiently encode paths forward of the animal at a decision point. Journal of Neuroscience 27:12176-12189.

Jouvet M (1969) Biogenic Amines and the States of Sleep. Science 163:32-41.

Karlsson MP, Frank LM (2009) Awake replay of remote experiences in the hippocampus. Nat Neurosci 12:913-918.

Kay K, Sosa M, Chung JE, Karlsson MP, Larkin MC, Frank LM (2016) A hippocampal network for spatial coding during immobility and sleep. Nature 531:185-190.

Kay K, Chung JE, Sosa M, Schor JS, Karlsson MP, Larkin MC, Liu DF, Frank LM (2020) Constant Sub-second Cycling between Representations of Possible Futures in the Hippocampus. Cell 180:552-567 e525.

Kloosterman F, Layton SP, Chen Z, Wilson MA (2014) Bayesian decoding using unsorted spikes in the rat hippocampus.217-227.

Lee AK, Wilson MA (2002) Memory of sequential experience in the hippocampus during slow wave sleep. Neuron 36:1183-1194.

Li W, Ma L, Yang G, Gan W-B (2017) REM sleep selectively prunes and maintains new synapses in development and learning. Nature Neuroscience 20:427-437. 
Louie K, Wilson MA (2001) Temporally structured replay of awake hippocampal ensemble activity during rapid eye movement sleep. Neuron 29:145-156.

Maharjan DM, Dai YY, Glantz EH, Jadhav SP (2018) Disruption of dorsal hippocampal - prefrontal interactions using chemogenetic inactivation impairs spatial learning. Neurobiol Learn Mem 155:351-360.

Masurkar AV, Srinivas KV, Brann DH, Warren R, Lowes DC, Siegelbaum SA (2017) Medial and Lateral Entorhinal Cortex Differentially Excite Deep versus Superficial CA1 Pyramidal Neurons. Cell reports 18:148-160.

McInnes L, Healy J, Melville J (2018) UMAP: Uniform Manifold Approximation and Projection for Dimension Reduction. arXiv preprint arXiv:180203426.

Mendelson WB, Bergmann BM (1999) Age-related Changes in Sleep in the Rat. Sleep 22:145-150.

Mizumori SJY, Perez GM, Alvarado MC, Barnes CA, McNaughton BL (1990) Reversible inactivation of the medial septum differentially affects two forms of learning in rats. Brain Research 528:12-20.

Mizuseki K, Miyawaki H (2017) Hippocampal information processing across sleep/wake cycles. Neurosci Res 118:30-47.

Mizuseki K, Diba K, Pastalkova E, Buzsáki G (2011) Hippocampal CA1 pyramidal cells form functionally distinct sublayers. Nat Neurosci 14:1174-1181.

Montgomery SM, Sirota A, Buzsáki G (2008) Theta and Gamma Coordination of Hippocampal Networks during Waking and Rapid Eye Movement Sleep. Journal of Neuroscience 28:6731-6741.

Morel P (2018) Gramm: grammar of graphics plotting in Matlab. Journal of Open Source Software 3:568.

Moser El, Kropff E, Moser MB (2008) Place Cells, Grid Cells, and the Brain's Spatial Representation System. AnnuRevNeurosci \%19;

Muessig L, Lasek M, Varsavsky I, Cacucci F, Wills TJ (2019) Coordinated Emergence of Hippocampal Replay and Theta Sequences during Post-natal Development. Current Biology 29:834-840.e834.

Müller C, Remy S (2017) Septo-hippocampal interaction. Cell and Tissue Research 373:565-575.

Müller GE, Pilzecker A (1900) Experimentelle beiträge zur lehre vom gedächtniss: JA Barth.

O'Keefe J (1976) Place units in the hippocampus of the freely moving rat. Experimental Neurology 51:78109.

O'Keefe J, Recce ML (1993) Phase relationship between hippocampal place units and the EEG theta rhythm. Hippocampus 3:317-330.

Oliva A, Fernández-Ruiz A, Buzsáki G, Berényi A (2016a) Role of Hippocampal CA2 Region in Triggering Sharp-Wave Ripples. Neuron 91:1342-1355.

Oliva A, Fernández-Ruiz A, Buzsáki G, Berényi A (2016b) Spatial coding and physiological properties of hippocampal neurons in the Cornu Ammonis subregions: SPATIAL CODING AND PHYSIOLOGICAL PROPERTIES OF HIPPOCAMPAL NEURONS. Hippocampus 26:1593-1607.

Orr G, Rao G, Houston FP, McNaughton BL, Barnes CA (2001) Hippocampal synaptic plasticity is modulated by theta rhythm in the fascia dentata of adult and aged freely behaving rats. Hippocampus 11:647-654.

Papale AE, Zielinski MC, Frank LM, Jadhav SP, Redish A (2016) Interplay between Hippocampal SharpWave-Ripple Events and Vicarious Trial and Error Behaviors in Decision Making. Neuron 92:975982.

Pastalkova E, Itskov V, Amarasingham A, Buzsáki G (2008) Internally generated cell assembly sequences in the rat hippocampus. Science 321:1322-1327.

Pavlides C, Greenstein YJ, Grudman M, Winson J (1988) Long-term potentiation in the dentate gyrus is induced preferentially on the positive phase of $\theta$-rhythm. Brain Research 439:383-387.

Petsche H, Stumpf C, Gogolak G (1962) The significance of the rabbit's septum as a relay station between the midbrain and the hippocampus I. The control of hippocampus arousal activity by the septum cells. Electroencephalography and Clinical Neurophysiology 14:202-211. 
Peyrache A, Lacroix MM, Petersen PC, Buzsáki G (2015) Internally organized mechanisms of the head direction sense. Nature neuroscience 18:569-575.

Pfeiffer BE, Foster DJ (2013) Hippocampal place-cell sequences depict future paths to remembered goals. Nature 497:74-79.

Poe GR (2017) Sleep Is for Forgetting. The Journal of Neuroscience 37:464-473.

Poe GR, Nitz DA, McNaughton BL, Barnes CA (2000) Experience-dependent phase-reversal of hippocampal neuron firing during REM sleep. Brain Res 855:176-180.

Rasch B, Born J (2013) About sleep's role in memory. Physiol Rev 93:681-766.

Ravassard P, Kees A, Willers B, Ho D, Aharoni D, Cushman J, Aghajan ZM, Mehta MR (2013) Multisensory Control of Hippocampal Spatiotemporal Selectivity. Science 340:1342-1346.

Redish AD (2016) Vicarious trial and error. Nat Rev Neurosci 17:147-159.

Samanta A, Alonso A, Genzel L (2020) Memory reactivations and consolidation: considering neuromodulators across wake and sleep. Current Opinion in Physiology 15:120-127.

Schmidt B, Duin AA, Redish AD (2019) Disrupting the medial prefrontal cortex alters hippocampal sequences during deliberative decision making. Journal of neurophysiology 121:1981-2000.

Schmidt R, Diba K, Leibold C, Schmitz D, Buzsaki G, Kempter R (2009) Single-trial phase precession in the hippocampus. Journal of Neuroscience 29:13232-13241.

Shin JD, Tang W, Jadhav SP (2019) Dynamics of Awake Hippocampal-Prefrontal Replay for Spatial Learning and Memory-Guided Decision Making. Neuron 104:1110-1125.e1117.

Siegle JH, Wilson MA (2014) Enhancement of encoding and retrieval functions through theta phasespecific manipulation of hippocampus. elife 3:e03061.

Silva D, Feng T, Foster DJ (2015) Trajectory events across hippocampal place cells require previous experience. Nat Neurosci 18:1772-1779.

Skaggs WE, McNaughton BL (1996) Replay of neuronal firing sequences in rat hippocampus during sleep following spatial experience. Science 271:1870-1873.

Skaggs WE, McNaughton BL, Wilson MA, Barnes CA (1996) Theta phase precession in hippocampal neuronal populations and the compression of temporal sequences. Hippocampus 6:149-172.

Squire LR, Genzel L, Wixted JT, Morris RG (2015) Memory Consolidation. Cold Spring Harbor Perspectives in Biology 7:a021766.

Tang W, Shin JD, Frank LM, Jadhav SP (2017) Hippocampal-prefrontal reactivation during learning is stronger in awake compared with sleep states. J Neurosci 37:11789-11805.

Thyng K, Greene C, Hetland R, Zimmerle H, DiMarco S (2016) True Colors of Oceanography: Guidelines for Effective and Accurate Colormap Selection. Oceanography 29:9-13.

Tingley D, Alexander AS, Quinn LK, Chiba AA, Nitz D (2018) Multiplexed oscillations and phase rate coding in the basal forebrain. Science Advances 4:eaar3230.

Twyver HV (1969) Sleep patterns of five rodent species. Physiology \& Behavior 4:901-905.

Valero M, Cid E, Averkin RG, Aguilar J, Sanchez-Aguilera A, Viney TJ, Gomez-Dominguez D, Bellistri E, Prida LMdl (2015) Determinants of different deep and superficial CA1 pyramidal cell dynamics during sharp-wave ripples. Nature Neuroscience 18:1281-1290.

Vanderwolf CH (1969) Hippocampal electrical activity and voluntary movement in the rat. Electroencephalogr Clin Neurophysiol 26:407-418.

Wang M, Foster DJ, Pfeiffer BE (2020) Alternating sequences of future and past behavior encoded within hippocampal theta oscillations. Science 370:247-250.

Wikenheiser AM, Redish AD (2013) The balance of forward and backward hippocampal sequences shifts across behavioral states. Hippocampus 23:22-29.

Wikenheiser AM, Redish A (2015a) Hippocampal theta sequences reflect current goals. Nat Neurosci 18:289-294. 
1368 Wikenheiser AM, Redish A (2015b) Decoding the cognitive map: ensemble hippocampal sequences and decision making. Curr Opin Neurobiol 32:8-15.

Winson J (1978) Loss of hippocampal theta rhythm results in spatial memory deficit in the rat. Science 201:160-163.

Wu C-T, Haggerty D, Kemere C, Ji D (2017) Hippocampal awake replay in fear memory retrieval. Nat Neurosci 20:571-580.

Xu H, Baracskay P, O’Neill J, Csicsvari J (2019) Assembly Responses of Hippocampal CA1 Place Cells Predict Learned Behavior in Goal-Directed Spatial Tasks on the Radial Eight-Arm Maze. Neuron 101:119-132.e114.

1378

1379

1380

1381

Zhang K, Ginzburg I, McNaughton BL, Sejnowski TJ (1998) Interpreting neuronal population activity by reconstruction: unified framework with application to hippocampal place cells. JNeurophysiol 79:1017-1044.

Zheng C, Bieri KW, Hsiao Y-T, Colgin LL (2016) Spatial Sequence Coding Differs during Slow and Fast Gamma Rhythms in the Hippocampus. Neuron 89:398-408. 
A

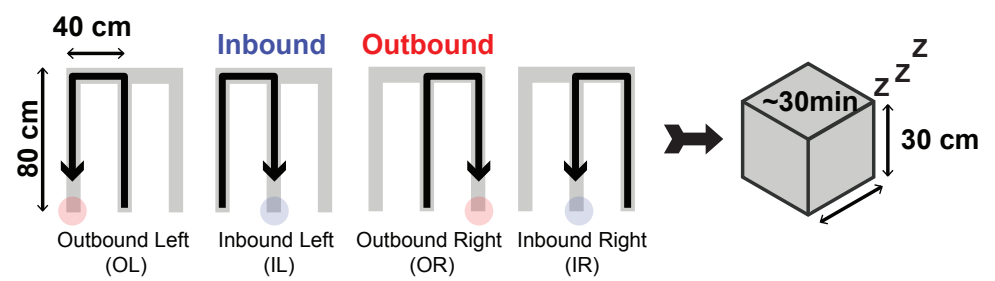

\section{B}
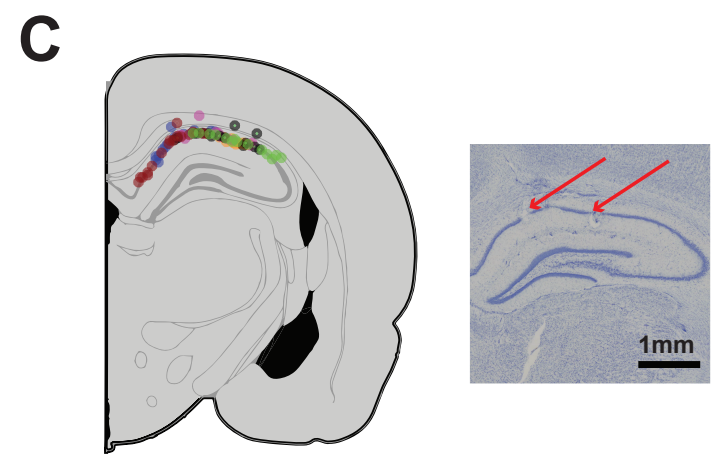

D
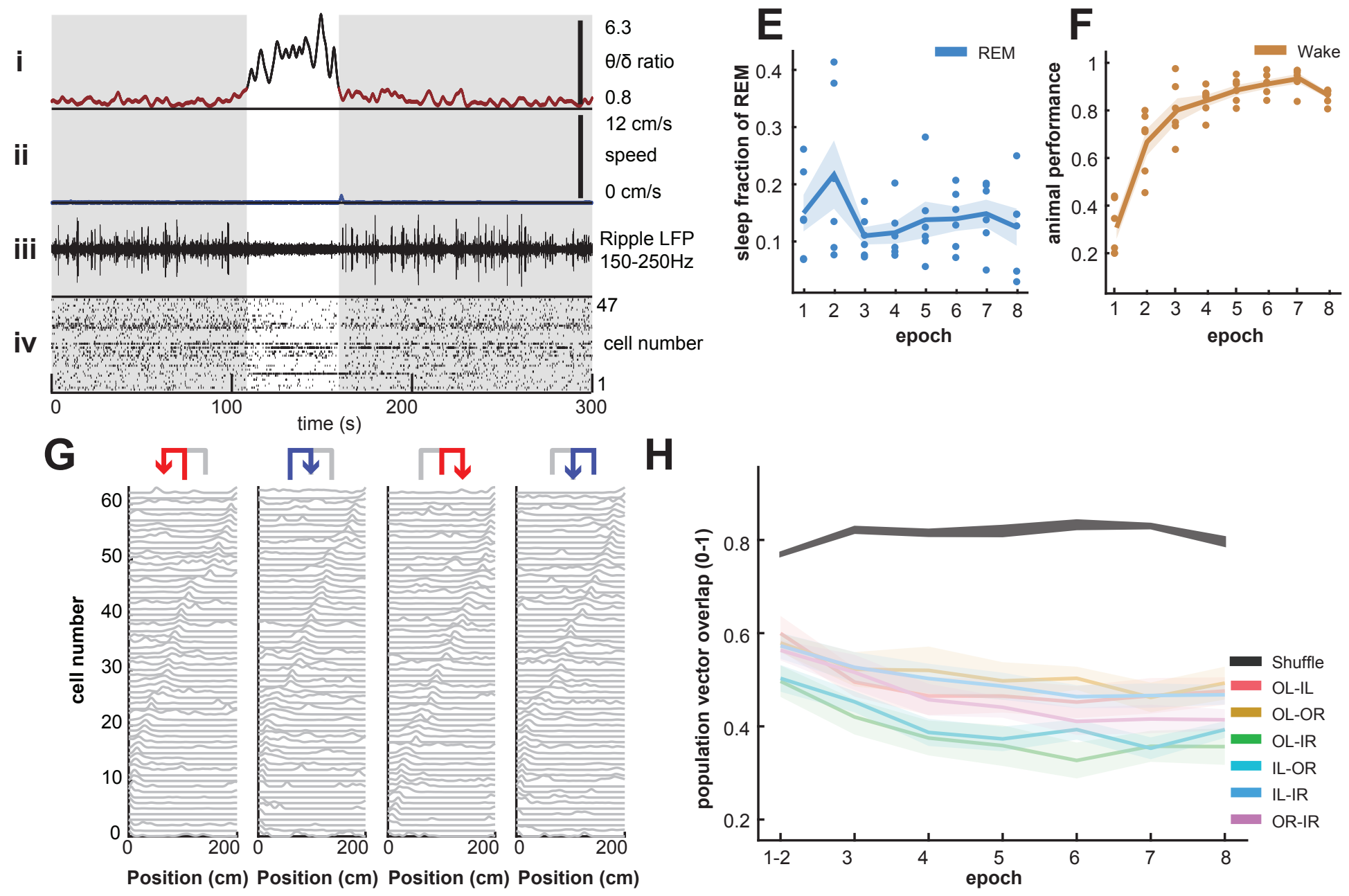

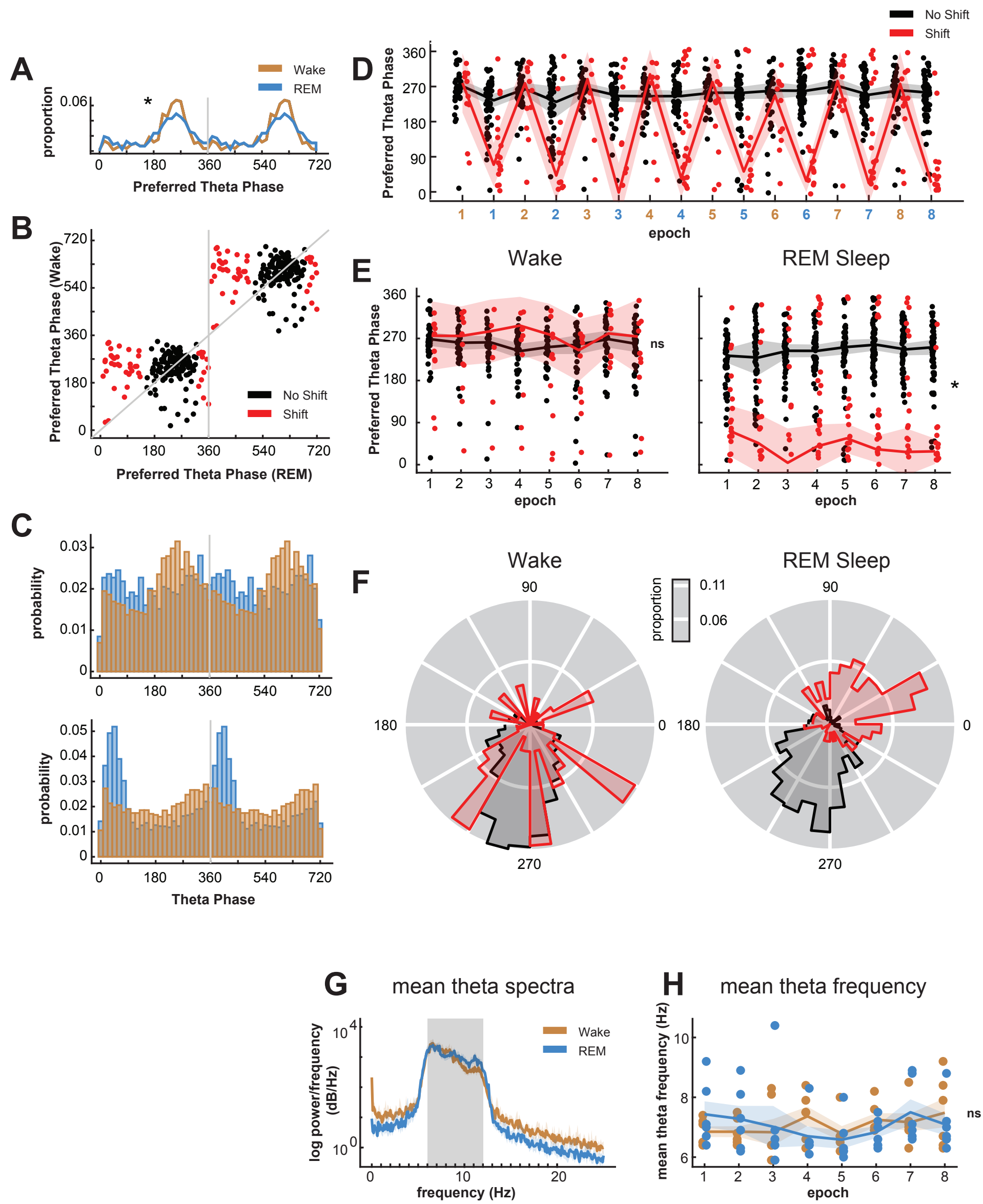


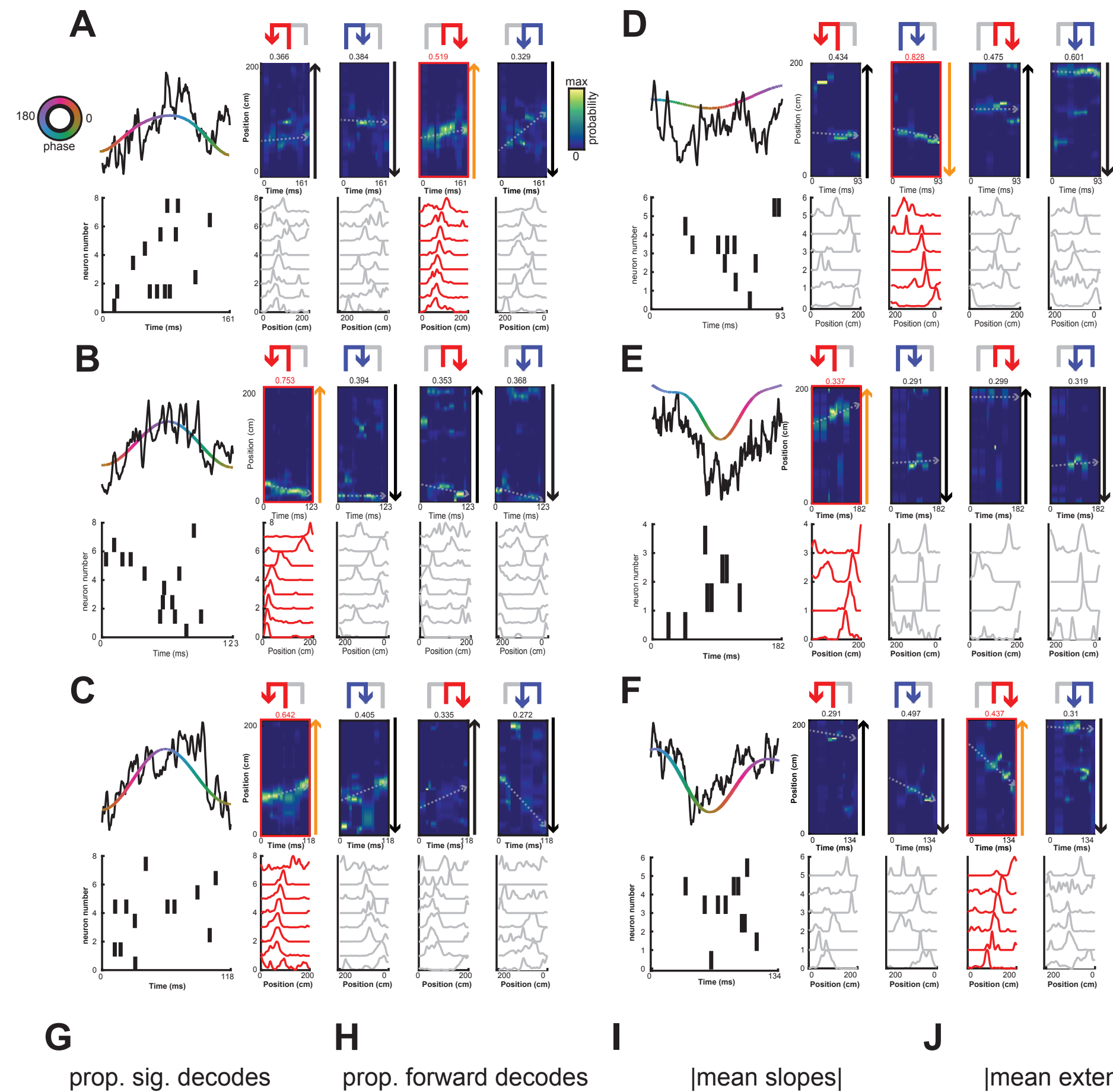

prop. sig. decodes prop. forward decodes
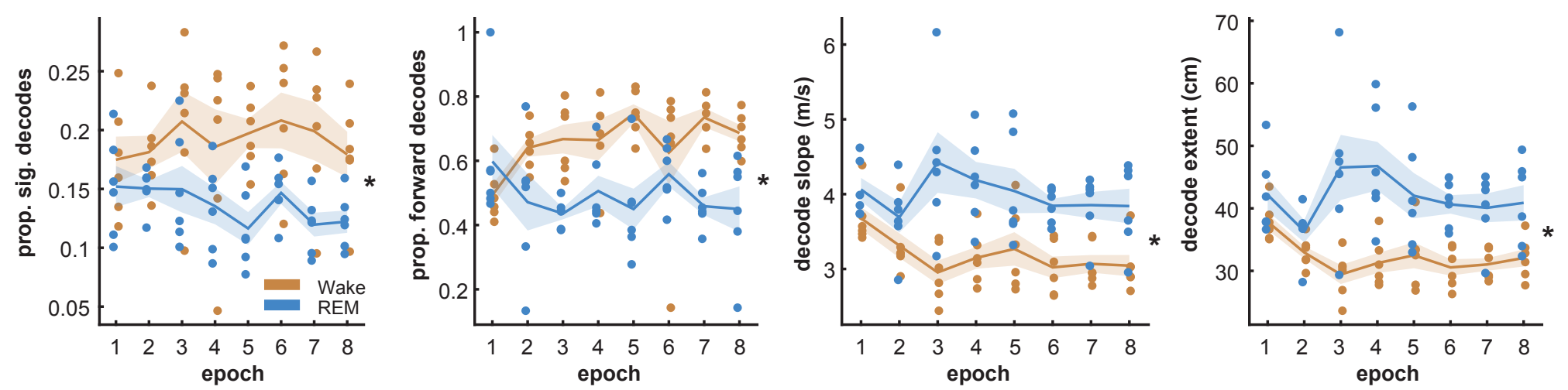
bioRxiv preprint doi: https://doi.org/10.1101/2021.04.15.439854; this version posted April 16, 2021. The copyright holder for this preprint (which

A
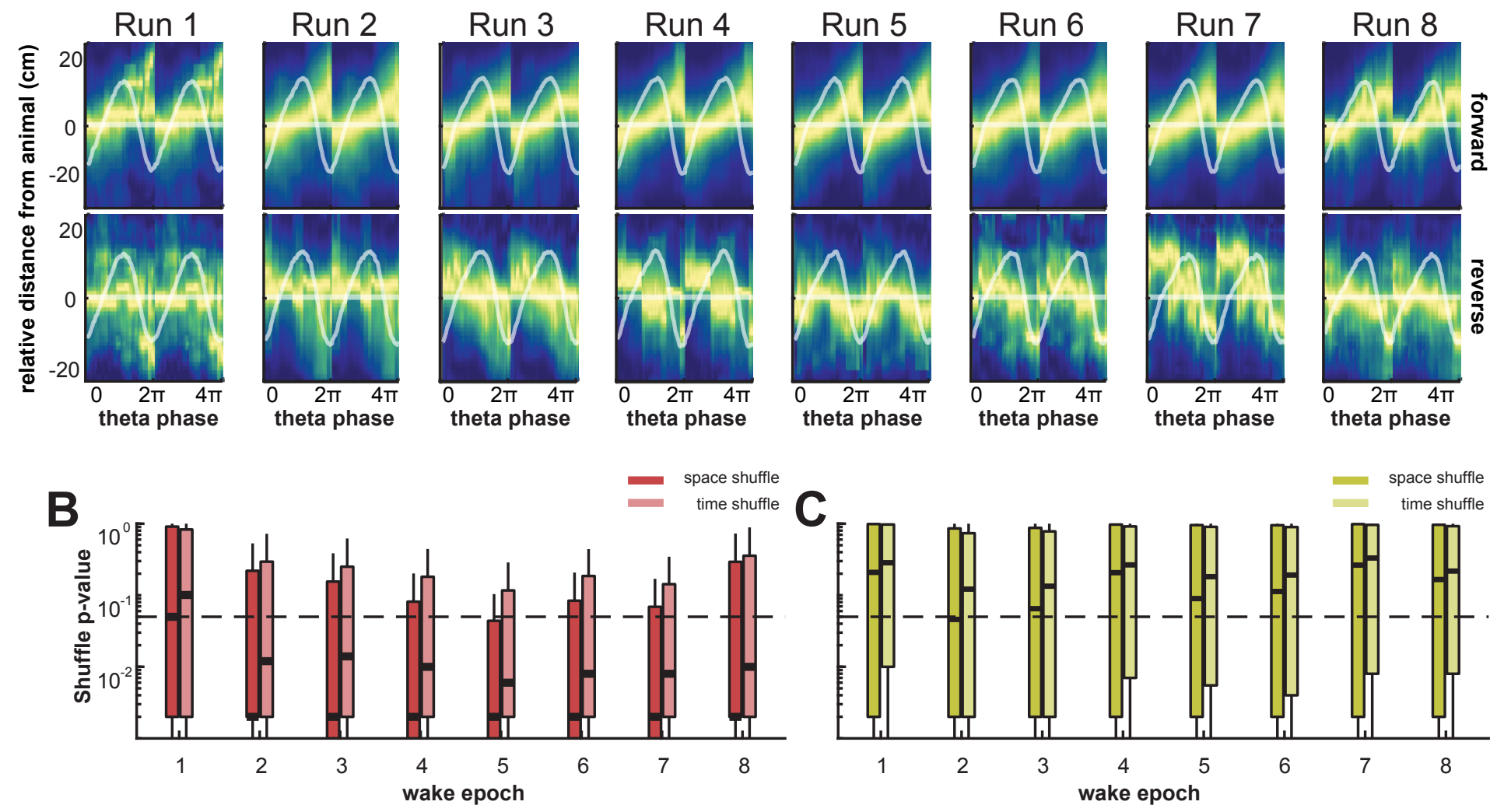

D
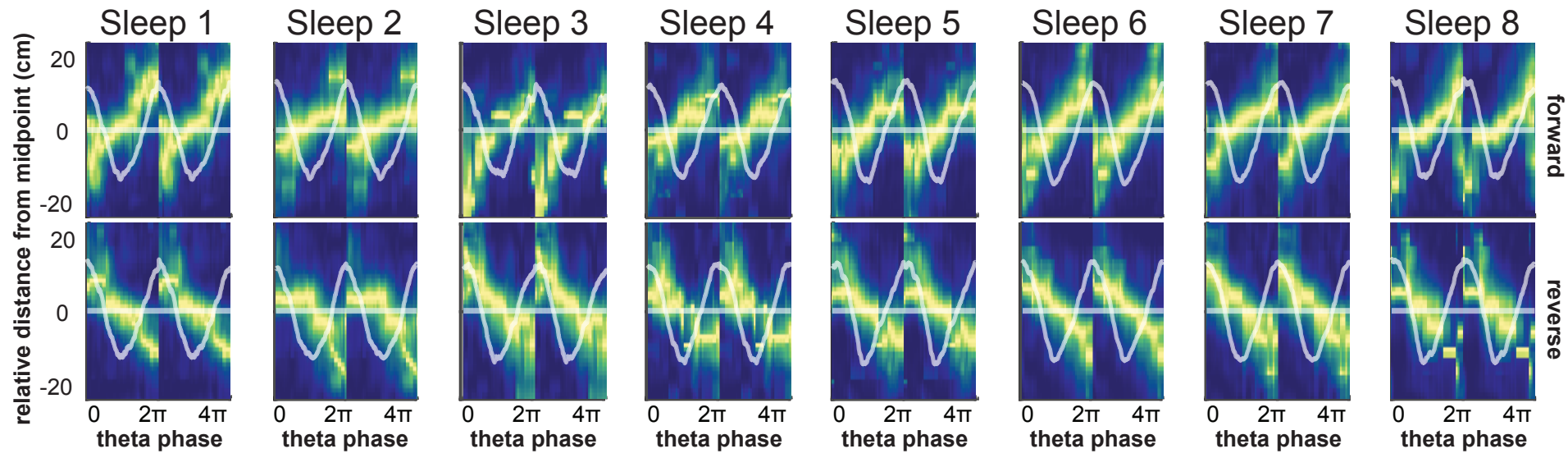

E

F
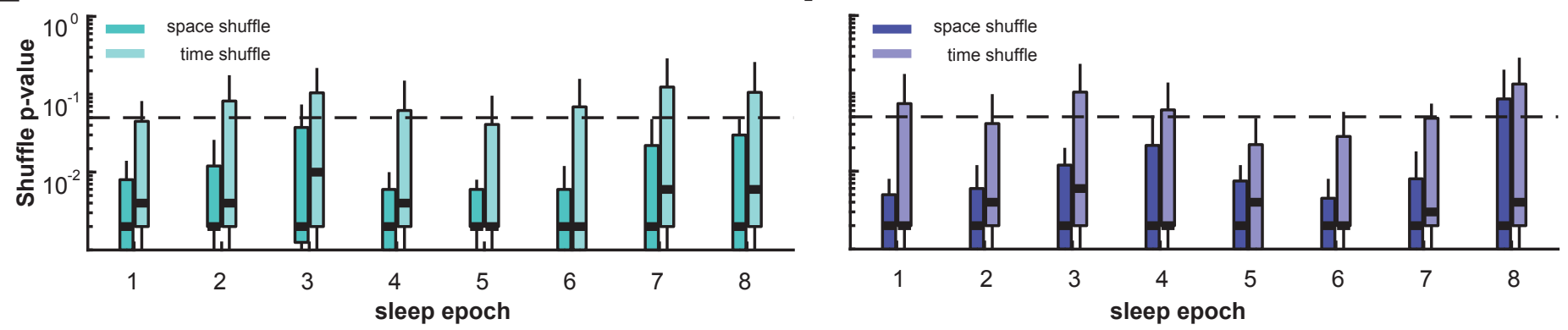
bioRxiv preprint doi: https://doi.org/10.1101/2021.04.15.439854; this version posted April 16, 2021. The copyright holder for this preprint (which was not certified by peer review) is the author/funder. All rights reserved. No reuse allowed without permission.
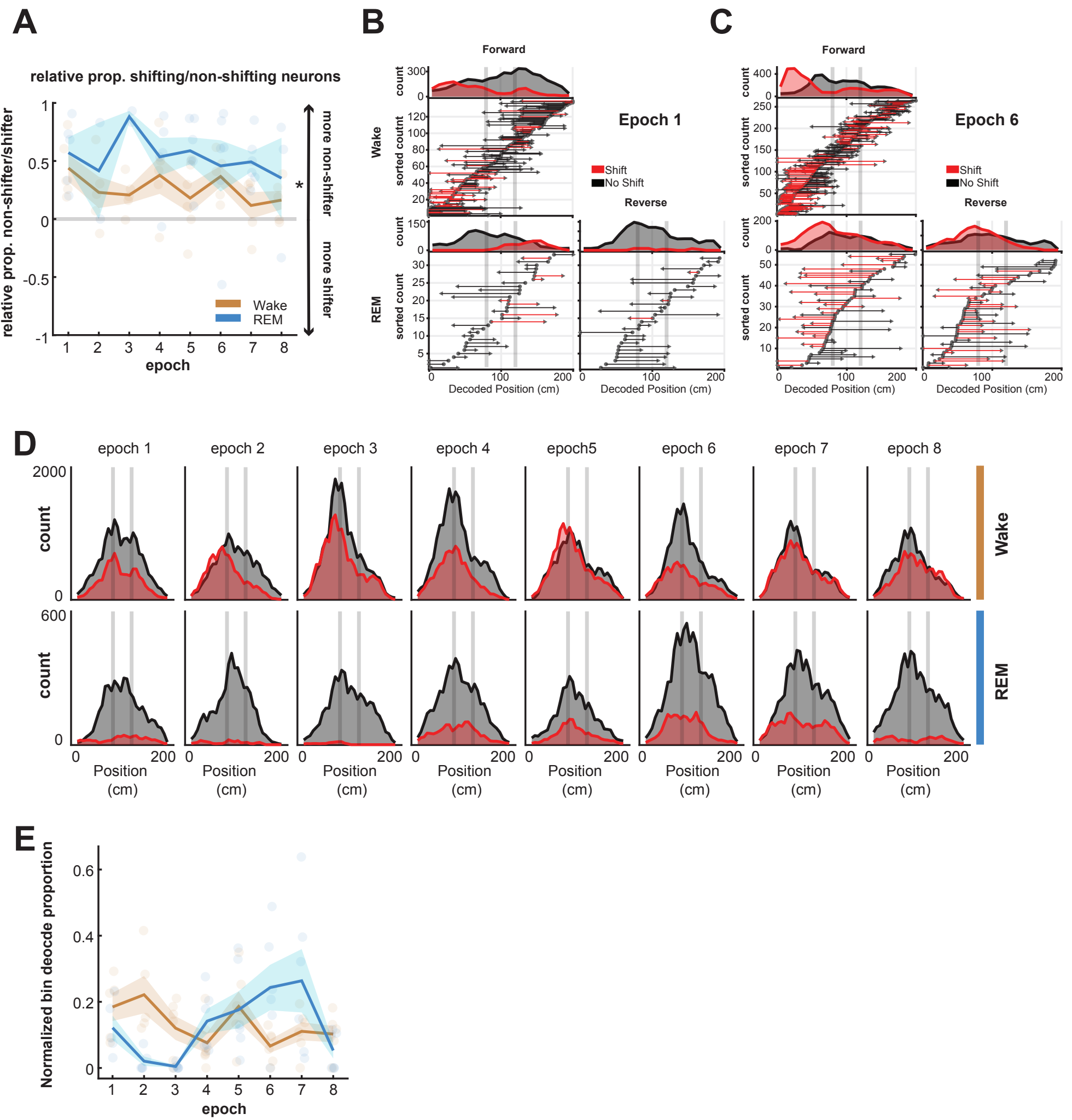
bioRxiv preprint doi: https://doi.org/10.1101/2021.04.15.439854; this version posted April 16, 2021. The copyright holder for this preprint (which was not certified by peer review) is the author/funder. All rights reserved. No reuse allowed without permission.

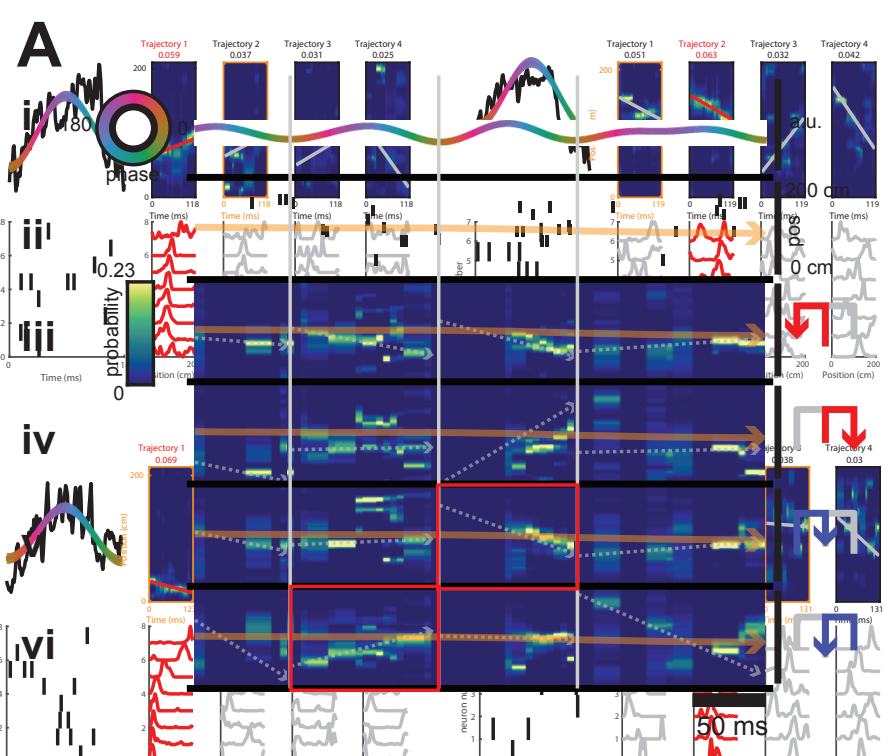

B
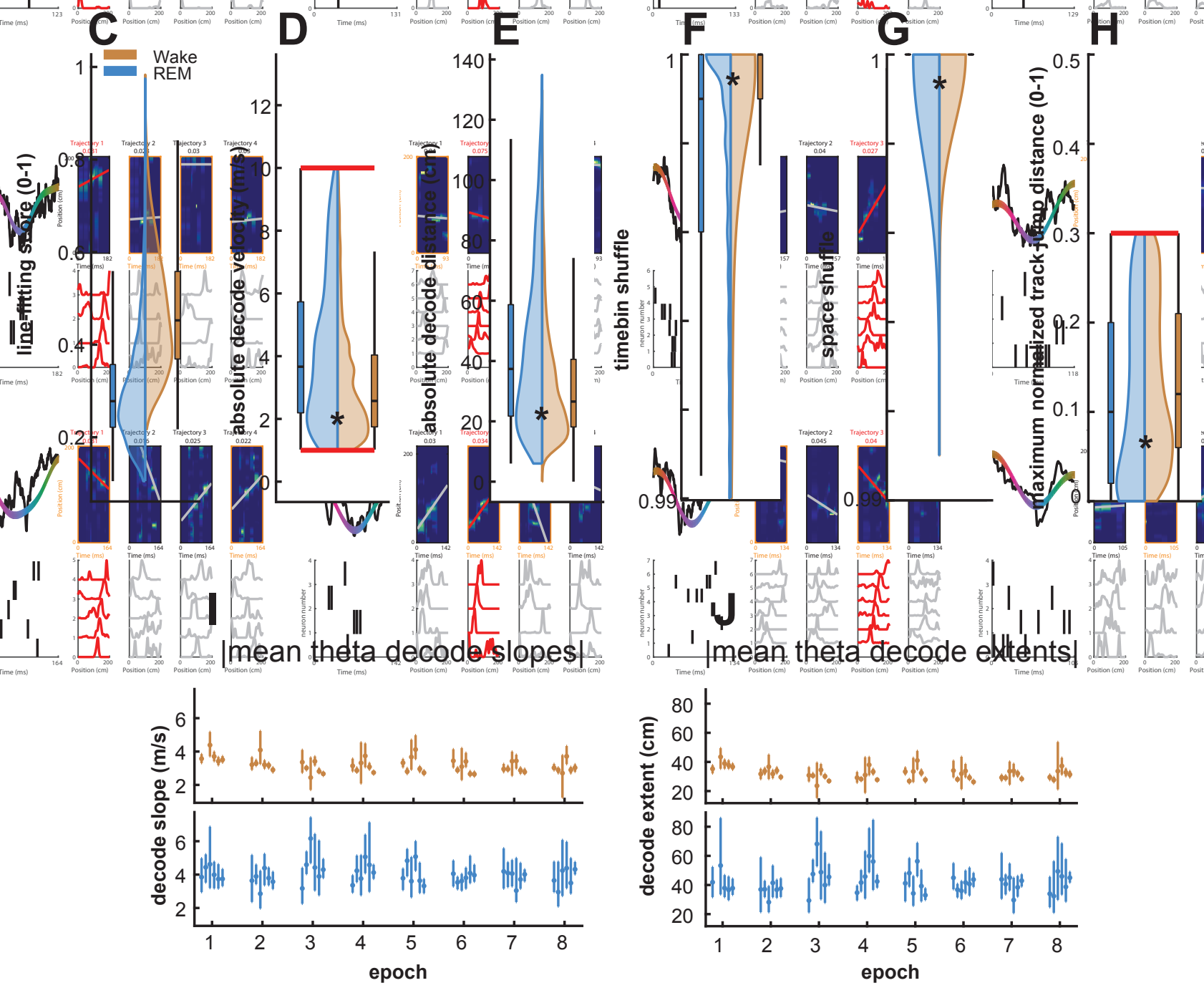
bioRxiv preprint doi: https://doi.org/10.1101/2021.04.15.439854; this version posted April 16, 2021. The copyright holder for this preprint (which was not certified by peer review) is the author/funder. All rights reserved. No reuse allowed without permission.

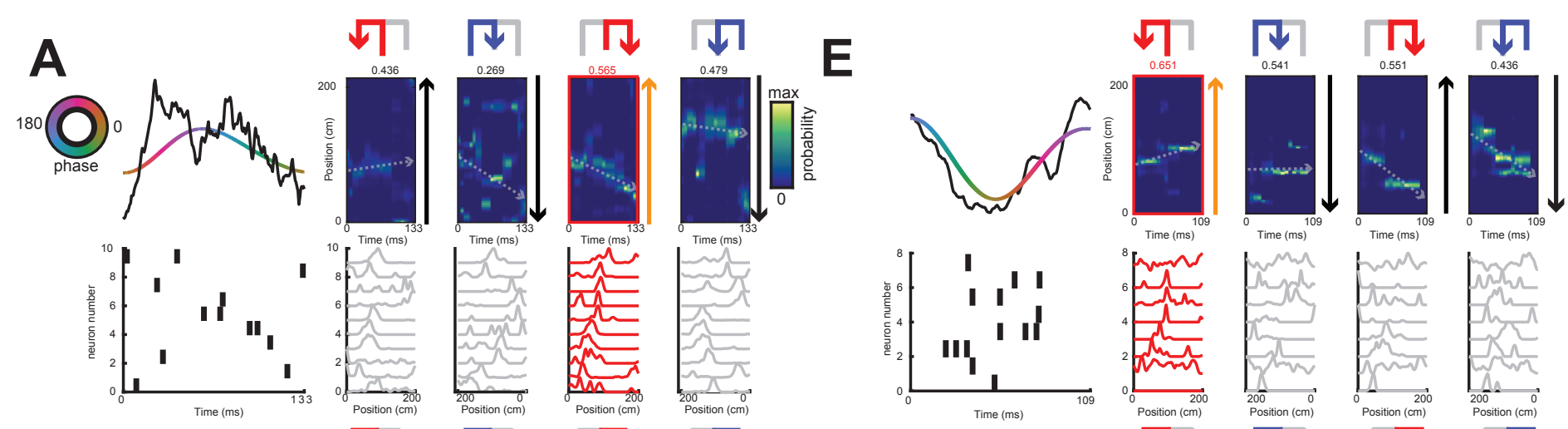

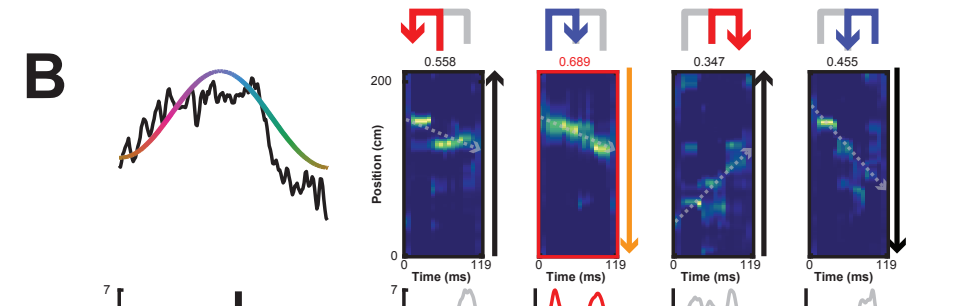
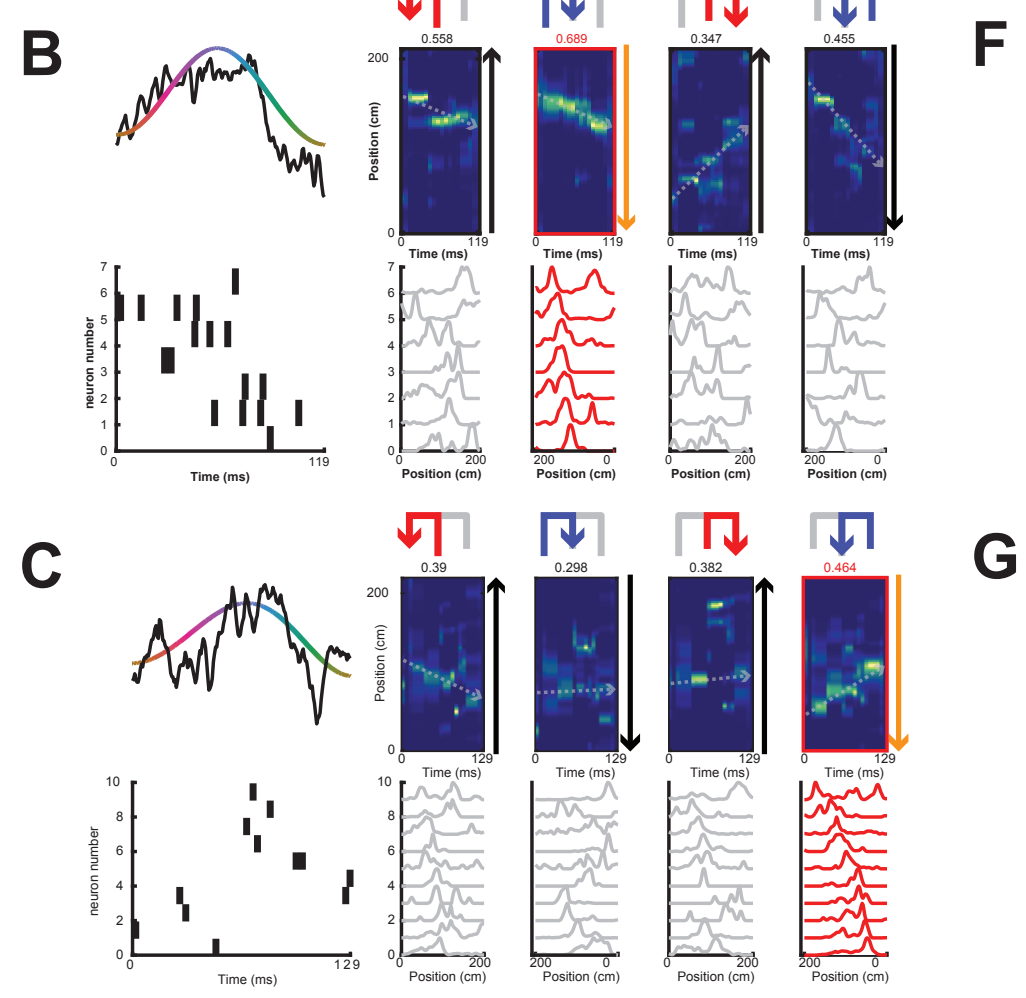

D
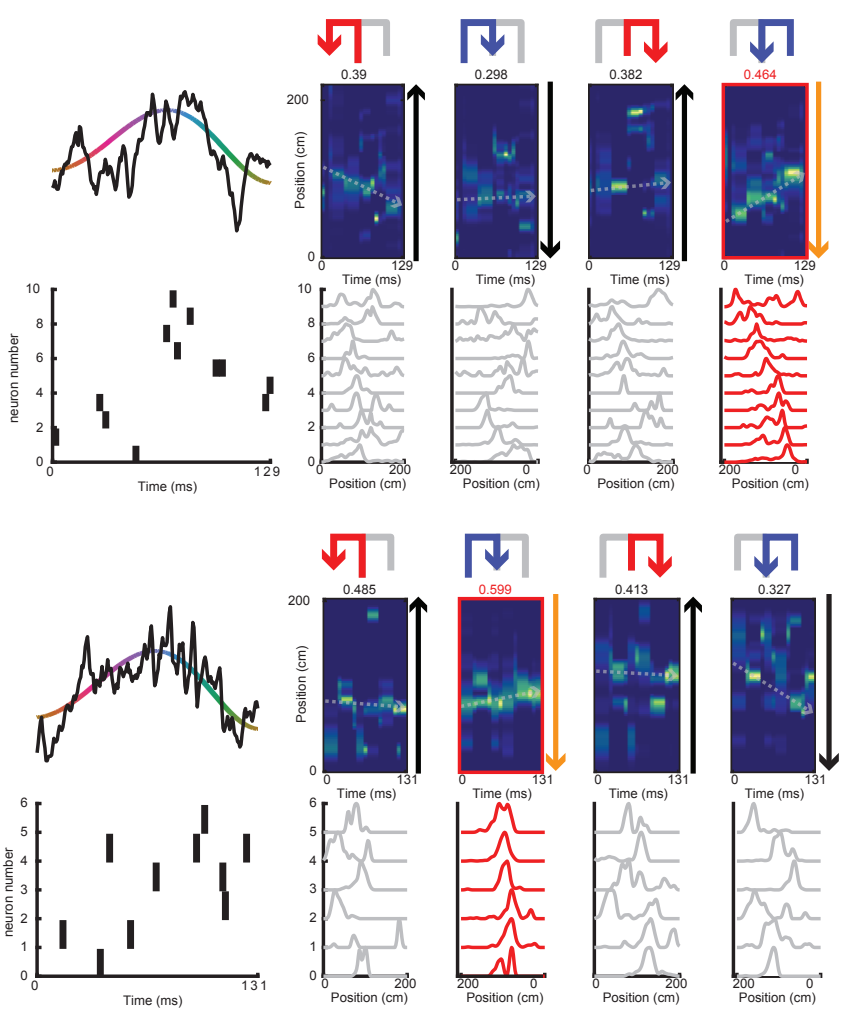

G

$\mathbf{H}$
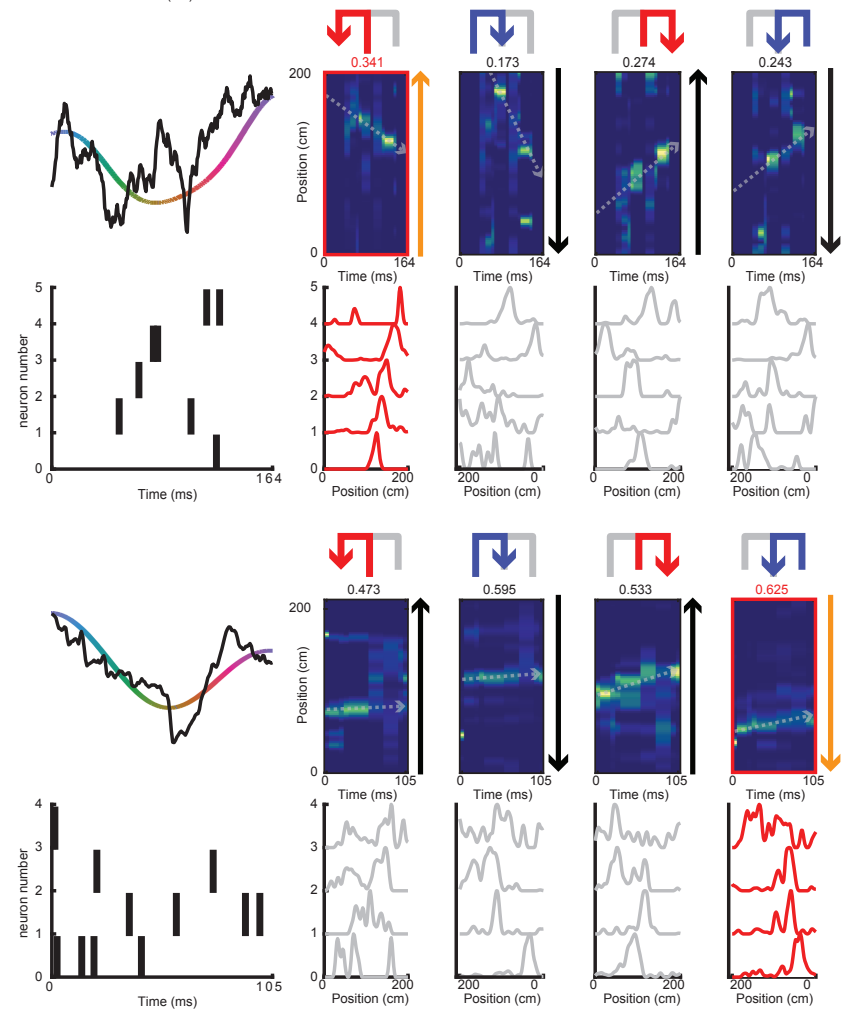

(n)
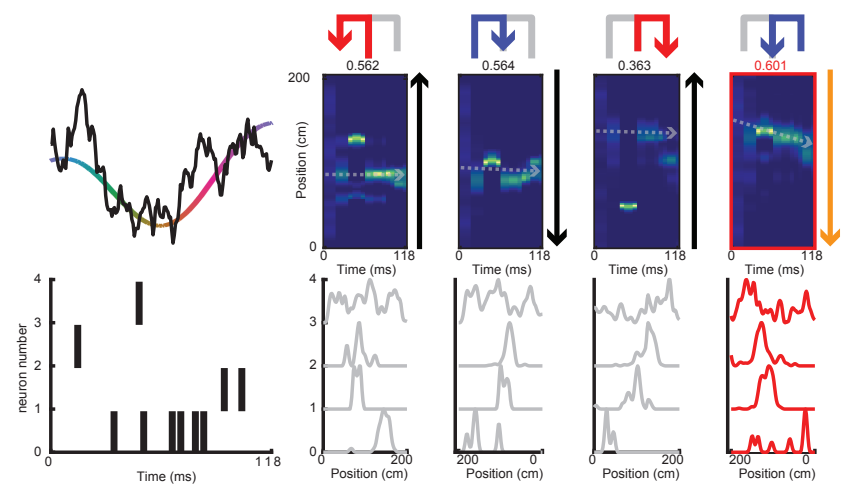
bioRxiv preprint doi: https://doi.org/10.1101/2021.04.15.439854; this version posted April 16, 2021. The copyright holder for this preprint (which was not certified by peer review) is the author/funder. All rights reserved. No reuse allowed without permission.

A

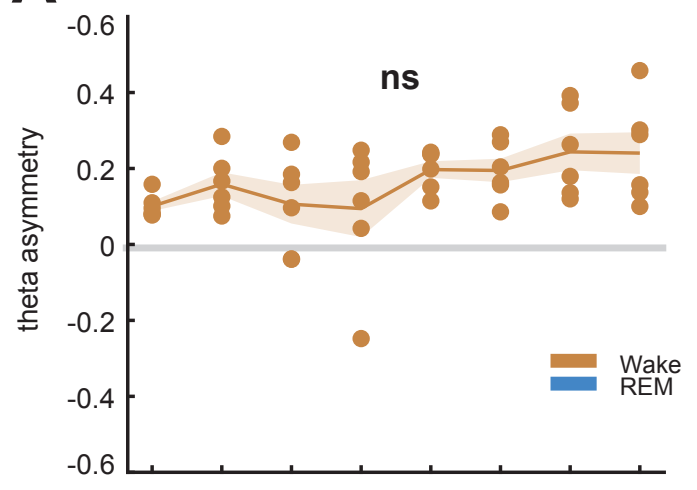

B

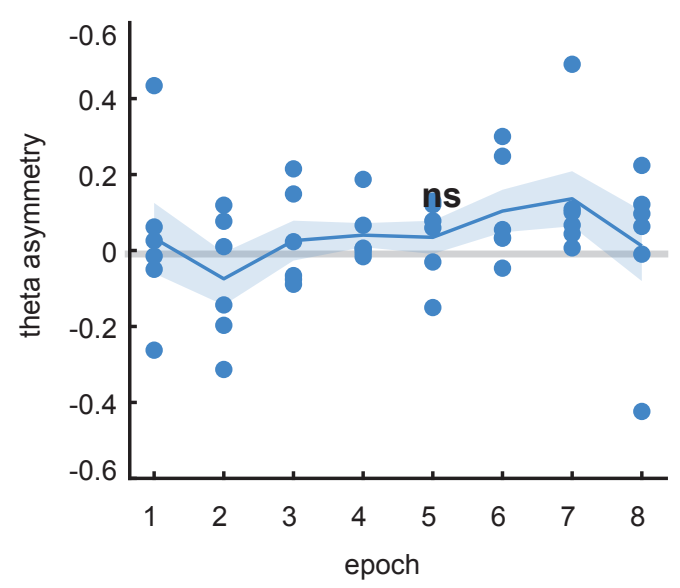

C

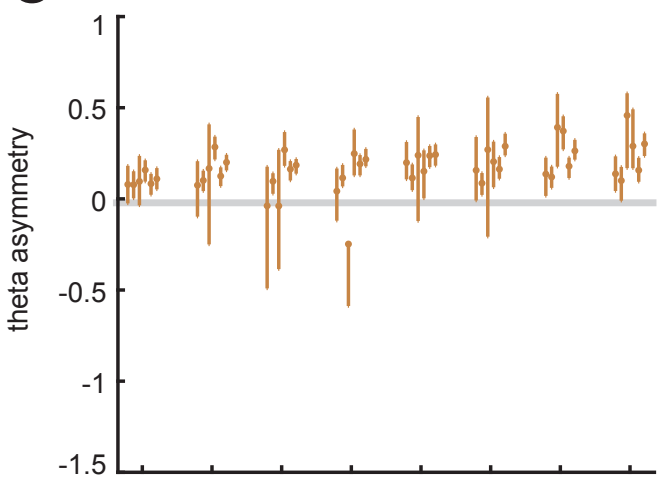

D

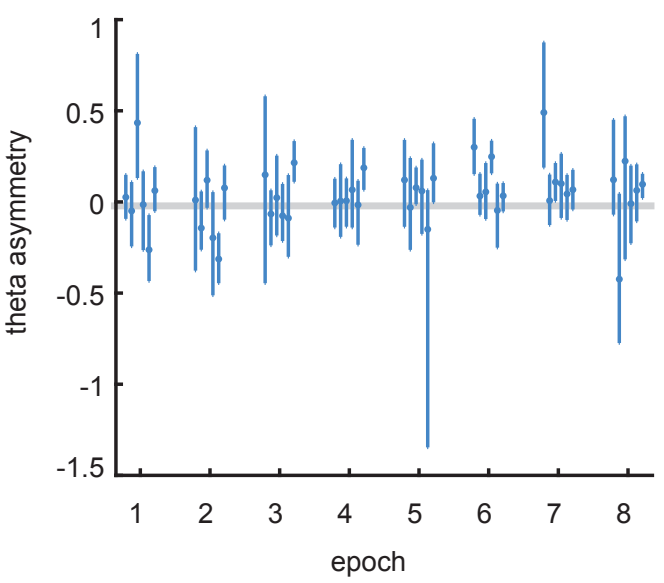


A
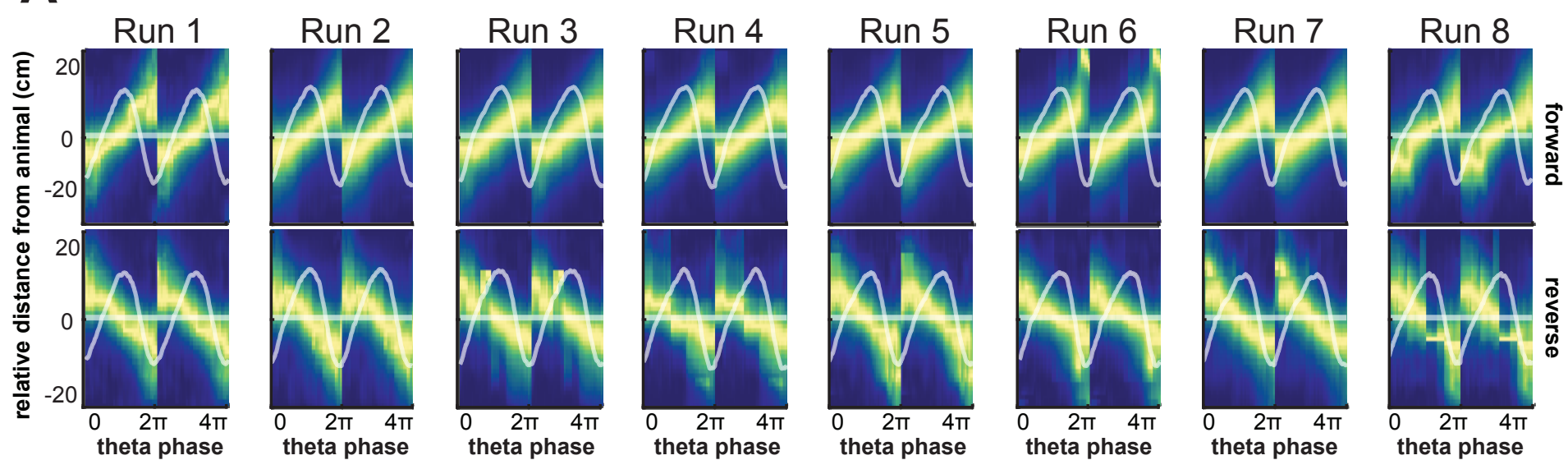

B

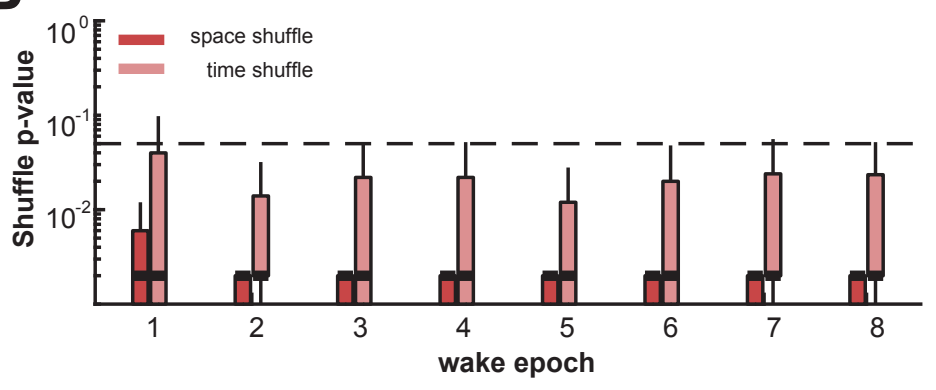

C

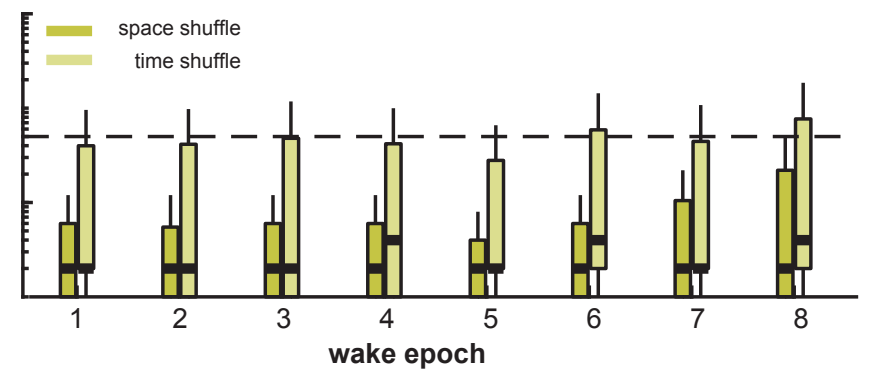

